\title{
HIGHER CODIMENSIONAL FOLIATIONS WITH KUPKA SINGULARITIES
}

\author{
O. CALVO-ANDRADE, M. CORRÊA JR, AND A. FERNÁNDEZ-PÉREZ
}

\begin{abstract}
We consider holomorphic foliations of dimension $k>1$ and codimension $\geq 1$ in the projective space $\mathbb{P}^{n}$, with a compact connected component of the Kupka set. We prove that, if the transversal type is linear with positive integers eigenvalues, then the foliation consist on the fibers of a rational fibration $\Phi: \mathbb{P}^{n} \rightarrow \mathbb{P}^{n-k}$. As a corollary, if $\mathcal{F}$ is a foliation such that $\operatorname{dim}(\mathcal{F}) \geq \operatorname{cod}(\mathcal{F})+2$ and has transversal type diagonal with different eigenvalues, then the Kupka component $K$ is a complete intersection and we get the same conclusion. The same conclusion holds if the Kupka set is a complete intersection and has radial transversal type. Finally, as an application, we find a normal form for non integrable codimension one distributions on $\mathbb{P}^{n}$.
\end{abstract}

\section{Contents}

1. Introduction.

1.1. Notation

1.2. Definitions

1.3. Statement of the results

2. The Kupka set.

2.1. Basic properties of the Kupka set

2.2. Geometry of the Kupka set.

2.3. The normal bundle of the Kupka set.

2.4. Normal Forms

3. Baum-Bott theory.

4. Holomorphic foliations on the projective space.

4.1. The projective space.

4.2. Foliations with a Kupka component

5. Rational Fibrations.

5.1. Geometrical Preliminary results

6. An application: Normal form of non-integrable codimension one distributions.

References

\section{INTRODUCTION.}

1.1. Notation. Let $M$ be a complex manifold. We denote by $\mathcal{O}_{M}, \Theta_{M}$ and $\Omega_{M}^{p}$ the sheaves of holomorphic functions, holomorphic vector fields and holomorphic $p$-forms over $M$. Therefore, if $n=\operatorname{dim}_{\mathbb{C}} M$, we denote by $\mathcal{K}_{M}=\Omega_{M}^{n}$ its canonical bundle $\left(\mathcal{O}_{n}, \Theta_{n}, \Omega_{n}^{p}\right.$ and $\mathcal{K}_{n}$ when $\left.M=\mathbb{P}^{n}\right)$. If $E$ is a holomorphic vector bundle,

Date: July 31, 2018.

2010 Mathematics Subject Classification. Primary 58A17- 32S65.

Key words and phrases. Kupka singular set - Holomorphic foliations. 
then $\Omega_{M}^{p}(E)$ denotes the sheaf of holomorphic $p$-forms with values in $E$. No distinction will be made between holomorphic vector bundles and locally free analytic sheaves.

1.2. Definitions. Let $U \subset \mathbb{C}^{n}$ be an open set and $1 \leq k<n$ an integer. A holomorphic $(n-k)$-form $\Omega \in \Omega_{U}^{n-k}(U)$ is integrable, if for each point $x \in U$, where $\Omega(x) \neq 0$, there exits a neighborhood $V$ of $x$ and holomorphic 1 -forms $\eta_{1}, \ldots, \eta_{n-k}$ such that

- $\left.\Omega\right|_{V}=\eta_{1} \wedge \cdots \wedge \eta_{n-k}$.

- There exists an $(n-k) \times(n-k)$ matrix of holomorphic 1 -forms $\theta=\left(\theta_{i j}\right)$ such that for all $i=1, \ldots, n-k$ the following equality holds.

$$
d \eta_{i}=\sum_{j=1}^{n-k} \theta_{i j} \wedge \eta_{j} .
$$

We recall the isomorphism between $\Theta_{U} \longleftrightarrow \Omega_{U}^{n-1}$ defined by the contraction by a vector field.

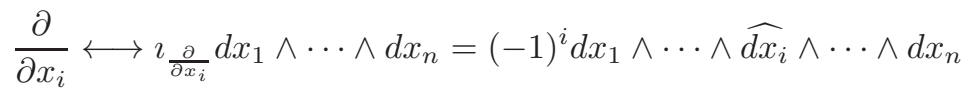

therefore, $(n-1)$-forms in $\mathbb{C}^{n}$ are always integrable.

Let $M$ be a complex manifold of dimension $n$ and $1 \leq k<n$. A holomorphic foliation $\mathcal{F}$ on $M$, of $\operatorname{dimension} \operatorname{dim}(\mathcal{F})=k$ and codimension $\operatorname{cod}(\mathcal{F})=n-k$, may be defined by a triple $\left\{\mathcal{U}=\left\{U_{\alpha}\right\}, \Omega_{\alpha}, \lambda_{\alpha \beta}\right\}$ where

(1) $\mathcal{U}=\left\{U_{\alpha}\right\}$ is an open covering of $M$.

(2) $\left\{\Omega_{\alpha}\right\}$ is a family of holomorphic, integrable $(n-k)$-forms, defined on the open set $U_{\alpha}$.

(3) If $U_{\alpha} \cap U_{\beta}:=U_{\alpha \beta} \neq \emptyset$, we have the family $\left\{\lambda_{\alpha \beta} \in \mathcal{O}^{*}\left(U_{\alpha \beta}\right)\right\}$ satisfying

$$
\left.\Omega_{\alpha}\right|_{U_{\alpha \beta}}=\left.\lambda_{\alpha \beta} \Omega_{\beta}\right|_{U_{\alpha \beta}}
$$

such that $\left\{\lambda_{\alpha \beta}\right\} \in \check{H}^{1}\left(\mathcal{U}, \mathcal{O}^{*}\right)$.

Let $\left\{W_{\sigma}, \Omega_{\sigma}, \mu_{\sigma \tau}\right\}$ be another triple satisfying conditions (1), (2) and (3) above. The triples $\left\{U_{\alpha}, \Omega_{\alpha}, \lambda_{\alpha \beta}\right\} \sim\left\{W_{\sigma}, \Omega_{\sigma}^{\prime}, \mu_{\sigma \tau}\right\}$ are equivalent if there exists a pair $\left\{\left(V_{i}, \rho_{i}\right)\right\}$, where $\left\{V_{i}\right\}$ is a refinement of the both coverings $\left\{W_{\sigma}\right\}$ and $\left\{U_{\alpha}\right\}$, and $\left\{\rho_{i} \in \mathcal{O}^{*}\left(V_{i}\right)\right\}$ is a family of functions such that if $V_{i} \subset U_{\alpha} \cap W_{\tau}$ then

$$
\left.\Omega_{\alpha}\right|_{V_{i}}=\left.\rho_{i} \cdot \Omega_{\tau}^{\prime}\right|_{V_{i}},
$$

it follows that $\lambda_{i j}=\rho_{i} \cdot \mu_{i j} \cdot \rho_{j}^{-1}$, and then $\left[\lambda_{\alpha \beta}\right]=\left[\mu_{\sigma \tau}\right] \in \check{H}^{1}\left(M, \mathcal{O}^{*}\right)$.

Also, for two equivalent triples $\left\{U_{\alpha}, \Omega_{\alpha}, \lambda_{\alpha \beta}\right\} \sim\left\{U_{\alpha}, \Omega_{\alpha}^{\prime}, \lambda_{\alpha \beta}\right\}$, the family of functions $\left\{U_{\alpha}, \rho_{\alpha}\right\}$ defines a global and never vanishing holomorphic function on $M$.

We denote by $L$ the holomorphic line bundle over $M$, represented by the 1cocycle $\left\{\lambda_{\alpha \beta}\right\} \in \check{H}^{1}\left(\mathcal{U}, \mathcal{O}^{*}\right)$. The family of $(n-k)$-forms $\left\{\Omega_{\alpha}\right\}$, glue in order to obtain a global holomorphic section $\left\{\Omega_{\alpha}\right\} \in \check{H}^{0}\left(\mathcal{U}, \Omega_{M}^{n-k}(L)\right)$. This motivates the following definition.

Definition 1.1. Let $M$ be a complex manifold of dimension $n$. Set $1 \leq k<n$. A holomorphic foliation $\mathcal{F}$ on $M$ of dimension $\operatorname{dim}(\mathcal{F})=k$ and $\operatorname{codimension} \operatorname{cod}(\mathcal{F})=$ $n-k$, is an equivalence class of integrable sections $[\Omega]$, where $\Omega \in H^{0}\left(M, \Omega^{n-k}(L)\right)$, and $L$ is a holomorphic line bundle over $M$.

The singular set of the foliation represented by the section $\Omega$, is defined by

$$
S(\mathcal{F})=S(\Omega):=\{p \in M \mid \Omega(p)=0\},
$$


it is an analytic set, with several irreducible components. We are going to denote by $S_{r}(\mathcal{F})$ the union of irreducible components of dimension $r$. We may assume always that the maximal dimension of the irreducible components of singular set is $n-2$.

We denote by $\mathcal{F}_{k}(M, L) \subset H^{0}\left(M, \Omega_{M}^{n-k}(L)\right)$, the set of equivalence classes integrable sections or foliations on $M$, in the case of the projective space, we use the notation $\mathcal{F}_{k}(n, c)$ instead $\mathcal{F}_{k}\left(\mathbb{P}^{n}, \mathcal{O}_{n}(c)\right)$. It is well known that, if $M$ is compact, $\mathcal{F}_{k}(M, L)$ is a projective variety in general, with several irreducible components.

Let $\mathcal{F} \in \mathcal{F}_{k}(M, L)$ be a foliation represented by the section $\Omega$. The tangent sheaf $T_{\mathcal{F}} \hookrightarrow \Theta$, is defined as follows: the stalk at any point $x \in M$ is

$$
\left(T_{\mathcal{F}}\right)_{x}=\left\{\mathbf{X} \in \Theta_{x} \mid \imath_{\mathbf{X}} \Omega=0\right\},
$$

the quotient $N_{\mathcal{F}}:=\Theta / T_{\mathcal{F}}$ is the normal sheaf of $\mathcal{F}$. Then, these sheaves are defined by the exact sequence

$$
0 \rightarrow T_{\mathcal{F}} \rightarrow \Theta \rightarrow N_{\mathcal{F}} \rightarrow 0
$$

We have that $T_{\mathcal{F}}$ is a coherent subsheaf of $\mathcal{O}_{M}$ modules, that is closed under the Lie bracket of vector fields. In some cases, it is locally free. The normal sheaf is also coherent, but it is not locally free over the singular set. The sequence (1.1) gives an alternative definition of holomorphic foliation [4.

The dual of $T_{\mathcal{F}}$ is the cotangent sheaf of $\mathcal{F}$. It will be denoted by $T^{*} \mathcal{F}$. Its determinant, i.e. $\operatorname{det}\left(T^{*} \mathcal{F}\right)=\left(\bigwedge^{k} T^{*} \mathcal{F}\right)^{* *}$ is the canonical bundle of the foliation, it will be denoted by $\mathcal{K}_{\mathcal{F}}=\Omega_{\mathcal{F}}^{k}$. Over the smooth locus $M \backslash S(\mathcal{F})$, the sheaves of the sequence (1.1) are locally free sheaves. Since the singular set has codimension at least two, we obtain the adjunction formula

$$
\mathcal{K}_{M}=\mathcal{K}_{\mathcal{F}} \otimes \operatorname{det} N_{\mathcal{F}}^{*}=\mathcal{K}_{\mathcal{F}} \otimes L^{*} \quad \text { Then } \quad \mathcal{K}_{\mathcal{F}}=\left(L \otimes \mathcal{K}_{M}\right)
$$

We see that the holomorphic line bundle $L$ correspond to the determinant line bundle of the normal sheaf $\operatorname{det}\left(N_{\mathcal{F}}\right)$.

After the dimension and the codimension of a holomorphic foliation, the main discrete invariant, is the first Chern class of the normal sheaf $N_{\mathcal{F}}$, and denoted by $c_{1}\left(N_{\mathcal{F}}\right)$. We define

$$
\mathcal{F} \in \mathcal{F}_{k}(M, L) \quad \text { then } \quad c_{1}\left(N_{\mathcal{F}}\right):=c_{1}(L) \in H^{2}(M, \mathbb{Z})
$$

For foliations on the projective space, we also define the degree of $\mathcal{F}$, it is the number $\operatorname{deg}(\mathcal{F})=c_{1}\left(N_{\mathcal{F}}\right)-\operatorname{cod}(\mathcal{F})-1$.

Definition 1.2. Let $\Omega \in H^{0}\left(M, \Omega^{n-k}(L)\right)$ be an integrable $(n-k)$-form.

(1) A holomorphic (meromorphic) integrating factor is a holomorphic (meromorphic) section $\varphi$ of the line bundle $L$ such that

$$
\eta=\frac{\Omega}{\varphi}
$$

is an integrable closed meromorphic form.

(2) A meromorphic function $f: M \rightarrow \mathbb{P}^{1}$ is a meromorphic first integral if

$$
\text { df } \wedge \Omega=0
$$

Observe that if $\varphi$ and $\psi$ are two linearly independent integrating factors of the foliation represented by $\Omega$, then the rational function $f=\varphi / \psi$ is a meromorphic first integral of $\Omega$.

Definition 1.3. The Kupka singular set of an integrable $(n-k)$-form $\Omega$ is defined by

$$
K(\mathcal{F})=K(\Omega):=\{p \in M \mid \Omega(p)=0, d \Omega(p) \neq 0\} \subset S(\mathcal{F})
$$


The singular and the Kupka sets, are well defined, they don't depend on the $(n-k)$-form $\Omega$ representing the foliation.

The Kupka set has the local product property (see Theorem 2.2): Given a foliation $\mathcal{F} \in \mathcal{F}_{k}(M, L)$, and a connected component $K \subset K(\mathcal{F}) \subset M$ of its Kupka set, there exists a unique germ of a foliation at $0 \in \mathbb{C}^{n-k+1}$, represented by a $(n-k)-$ form $\eta \in \Omega_{0}^{n-k}\left(\mathbb{C}^{n-k+1}\right)$ or equivalently a germ of a holomorphic vector field $\mathbf{X} \in$ $\Theta_{0}\left(\mathbb{C}^{n-k+1}\right)$, and related by the formula

$$
\eta=\imath_{\mathbf{X}} d x_{0} \wedge \cdots \wedge d x_{n-k}
$$

such that for each point $x \in K$, there exists a pair $(U, \phi)$ where $U \subset M$ is an open set and $\phi:(U, x) \rightarrow\left(\mathbb{C}^{n-k+1}, 0\right)$ is a submersion such that

(1) $\phi^{-1}(0)=K \cap U$, therefore $K$ is a smooth submanifold and $\operatorname{dim}(K)=$ $\operatorname{dim}(\mathcal{F})-1$.

(2) The $(n-k)$-form in $\phi^{*} \eta$ defines $\left.\mathcal{F}\right|_{U}$.

(3) The Kupka set is persistent under deformations of the foliation.

Locally, the foliation looks like $\mathcal{G}_{\mathbf{X}} \times \mathbb{C}^{k-1}$, the product of a singular foliation by curves in $\mathbb{C}^{n-k+1}$ defined by the solutions of $\mathbf{X}$ times a non singular holomorphic foliation of dimension $k-1$.

The set

$$
\mathcal{K}_{k}(M, L)=\left\{\mathcal{F} \in \mathcal{F}_{k}(M, L) \mid K(\mathcal{F}) \supset K \text { a compact connected component }\right\},
$$

is an open subset of $\mathcal{F}_{k}(M, L)$ and hence, its closure is a union of irreducible components.

An important problem in the theory of foliations, is the classification of the foliations $\mathcal{K}_{k}(M, L) \subset \mathcal{F}_{k}(M, L), \quad n \geq 3$. The codimension-one case i.e., when $1<\operatorname{dim}(\mathcal{F})=n-1$, for foliations on the projective space, was first considered by Cerveau and Lins Neto in 11. They proved the following Theorem:

Theorem 1.4. Let $\mathcal{F} \in \mathcal{K}_{n-1}(n, c), \quad n \geq 3$. If $K(\mathcal{F})$ is a complete intersection then the leaves of $\mathcal{F}$ are the fibers of a rational map $\Phi: \mathbb{P}^{n} \rightarrow \mathbb{P}^{1}$.

Furthermore, Cerveau and Lins Neto conjectured that $K(\mathcal{F})$ is always a complete intersection. Recently, Brunella [5] and Calvo-Andrade [6, 7], have given a positive answer about this conjecture. As consequence of these results, there is a complete classification of these foliations on the projective space $\mathbb{P}^{n}, \quad n \geq 3$.

1.3. Statement of the results. In this paper, we study the set of foliations $\mathcal{K}_{k}(M, L) \subset \mathcal{F}_{k}(M, L)$ of holomorphic foliations of dimension $1<k \leq n-1$ with a compact connected Kupka component in its singular set. We mainly assume that $1<k \leq n-2$.

Given a foliation $\mathcal{F} \in \mathcal{K}_{k}(M, L)$ with Kupka set $K=K(\mathcal{F})$. It is natural to ask about the relations between the invariants of the embedding $\jmath: K \hookrightarrow M$, mainly, the total Chern class of the normal bundle $c(\nu(K, M)) \in H^{*}(K, \mathbb{Z})$, and the invariants of the foliation.

For instance, Proposition 2.4 said that $\wedge^{k+1} \nu(K, M)=\left.L\right|_{K}$, and in particular

$$
c_{1}(\nu(K, M))=j^{*} c_{1}\left(N_{\mathcal{F}}\right) \in H^{2}(K, \mathbb{Z}),
$$

which implies that, if $L$ is an ample line bundle, the embedding $\jmath: K \hookrightarrow M$ is not topologically trivial and at least, the first Chern class of the normal bundle, is the restriction of a non zero class on $H^{2}(M, \mathbb{Z})$.

On the other hand, since the transversal vector field $\mathbf{X}$ has $\operatorname{Div}(\mathbf{X})_{0} \neq 0$, it has not trivial linear part $\mathbf{X}_{1}$. The Jordan decomposition of $\mathbf{X}_{1}$, implies that the normal bundle of a Kupka component, decomposes in a direct sum of subbundles, or it is indecomposable and projectively flat (Theorem 2.6). As we will see later, 
this property, imposes serious restrictions on the geometry of the embedding of Kupka set.

In section 3. we prove a deeper relation between the transversal vector field, the first Chern class of the normal sheaf of the foliation and the fundamental class of the Kupka set $\jmath(K) \subset M$.

The Theorem 3.3 of section 3 , states that, for a foliation $\mathcal{F} \in \mathcal{K}_{k}(M, L)$ with a compact connected component $K$ and transversal type $\mathbf{X}$ the fundamental class of $K \subset M$ satisfies the formula

$$
[K] \cdot \operatorname{Res}\left(c_{1}\left(J_{\mathbf{X}}\right)^{\operatorname{cod}(\mathcal{F})+1}, \mathbf{X}, 0\right)=c_{1}\left(N_{\mathcal{F}}\right)^{\operatorname{cod}(\mathcal{F})+1} .
$$

In the equation above, $\operatorname{Res}\left(c_{1}\left(J_{\mathbf{X}}\right)^{\operatorname{cod}(\mathcal{F})+1}, \mathbf{X}, 0\right)$ is the Baum-Bott residue of the transversal vector field and $[K]$ denotes the fundamental class of $K \subset M$.

Let $\mathcal{F}$ be a foliation with a Kupka component with linear transversal type

$$
\mathbf{X}\left(x_{0}, \ldots, x_{n-k}\right)=\sum_{i=0}^{n-k} \lambda_{i} x_{i} \frac{\partial}{\partial x_{i}}, \quad \text { with } \quad \lambda_{i} \in \mathbb{N} \quad \lambda_{i} \neq \lambda_{j}
$$

In section 2. we introduce the notion of resonance. If the transversal vector field is linear, and diagonal with positive integers eigenvalues, we find a meromorphic integrating factor $G$ for the transversal model $\Omega=\imath_{\mathbf{X}} d x_{0} \wedge \cdots \wedge d x_{n-k}$. We get the normal form:

$$
\frac{\Omega}{G}=\left(\sum_{j=0}^{\ell}(-1)^{i} \lambda_{i} \frac{d x_{0}}{x_{0}} \wedge \ldots \frac{\widehat{d x_{i}}}{x_{i}} \cdots \wedge \frac{d x_{\ell}}{x_{\ell}}\right) \wedge d \varphi_{\ell+1} \wedge \cdots \wedge d \varphi_{n-k},
$$

and a meromorphic function $F: U \rightarrow \mathbb{P}^{n-k}$, whose fibers are the leaves.

The subset $\Lambda_{N R}=\left\{\lambda_{0}<\cdots<\lambda_{\ell}\right\}$ is the maximal subset of non-resonant eigenvalues. On the other hand, for all $j=1, \ldots, n-k-\ell$, the set of eigenvalues $\Lambda_{R}=\left\{\lambda_{\ell+1}<\cdots<\lambda_{n-k}\right\}$, we may find resonance relations of the type

$$
\lambda_{\ell+j}=\lambda_{0} \cdot m_{0}^{\ell+j}+\cdots+\lambda_{\ell} \cdot m_{\ell}^{\ell+j} .
$$

We define the polynomial functions

$$
h^{\ell+j}\left(x_{0}, \ldots, x_{\ell}\right)=x_{0}^{m_{0}^{\ell+j}} \cdots x_{\ell}^{m_{\ell}^{\ell+j}}, \quad \varphi_{\ell+j}=\frac{x_{\ell+j}}{h^{\ell+j}\left(x_{0}, \ldots, x_{\ell}\right)} .
$$

With a more carefull work, the above formal may be written, on a neighborhood of the Kupka set as

$$
\frac{\Omega}{\widetilde{G}}=\left(\sum_{j=0}^{\ell}(-1)^{i} \lambda_{i} \frac{d x_{0}}{x_{0}} \wedge \ldots \frac{\widehat{d x_{i}}}{x_{i}} \cdots \wedge \frac{d x_{\ell}}{x_{\ell}}\right) \wedge \theta_{\ell+1} \wedge \cdots \wedge \theta_{n-k}, \quad d \theta_{j}=0 .
$$

With the use of (1.2), we prove the following generalization of the Theorem ([1], Theorem B]).

Theorem 1. Let $\mathcal{F} \in \mathcal{K}_{k}(n, c)$ be a holomorphic foliation with $S_{k-1}(\mathcal{F})=K(\mathcal{F})$ and transversal type

$$
\mathbf{X}=\sum_{i=0}^{n-k} \lambda_{i} x_{i} \frac{\partial}{\partial x_{i}}, \quad \lambda_{i} \in \mathbb{N} \quad \lambda_{i} \neq \lambda_{j}, \text { for all } i \neq j .
$$

Then the leaves of the foliation are the fibers of a rational fibration and it is represented by a $(n-k)$-form of the type

$$
\Omega\left(z_{0}, \ldots, z_{n}\right)=\sum_{i=0}^{n-k}(-1)^{i} \lambda_{i} \cdot f_{i}\left(z_{0}, \ldots, z_{n}\right) d f_{0} \wedge \ldots \wedge \widehat{d f_{i}} \wedge \ldots \wedge d f_{n-k} .
$$


We say that a foliation $\mathcal{F}$ has small codimension when

$$
\operatorname{dim}(\mathcal{F}) \geq \operatorname{cod}(\mathcal{F})+2 .
$$

We will see that this condition, we have strong restrictions on the geometry of the Kupka set and the possibilities of the transversal type.

Let $\mathcal{F} \in \mathcal{K}_{k}(n, c)$ be a foliation with small codimension, by Bezout's theorem, $S_{k-1}(\mathcal{F})=K(\mathcal{F})=K$ and it is compact and connected. Also, if the transversal type is linear with different eigenvalues and $n \geq 5$, according with [1, the inclusion map induces an isomorphism $\jmath^{*}: \operatorname{Pic}\left(\mathbb{P}^{n}\right) \stackrel{\sim}{\rightarrow} \operatorname{Pic}(K) \simeq \mathbb{Z}$. We obtain the following result.

Corollary 2. Let $\mathcal{F} \in \mathcal{K}_{k}(n, c)$ be a foliation of small codimension and linear transversal type

$$
\mathbf{X}=\sum_{i=0}^{k} \lambda_{i} x_{i} \frac{\partial}{\partial x_{i}} \quad\left(\lambda_{i}-\lambda_{j}\right) \neq 0 \neq \lambda_{j} \quad \text { for all } \quad i \neq j,
$$

then $\lambda_{i} \in \mathbb{N}$ and $\mathcal{F}$ is a rational fibration.

We also have a version for radial transversal type (2.20).

Theorem 3. Let $\mathcal{F} \in \mathcal{K}_{k}(n, c)$ with a Kupka set $K$ and radial transversal type. If $K$ is a complete intersection, then $\mathcal{F}$ is a rational fibration of the type

$$
\left[f_{0}: \cdots: f_{n-k}\right]: \mathbb{P}^{n} \rightarrow \mathbb{P}^{n-k}, \quad \operatorname{deg}\left(f_{j}\right)=\frac{c}{n-k+1}
$$

Finally, in section (6), we have an application to codimension one non integrable distributions $\mathcal{D}$.

Let $\omega$ be a germ of 1 -form on $\left(\mathbb{C}^{n}, 0\right)$. We denote by

$$
(d \omega)^{s}=\overbrace{d \omega \wedge \cdots \wedge d \omega}^{\text {s times }} .
$$

Let $\omega \in H^{0}\left(M, \Omega_{M}^{1}(L)\right)$. We define the class of the foliation induced by $\omega$ to be the integer $r$ for which generically

$$
\omega \wedge(d \omega)^{r-1} \neq 0, \quad \omega \wedge(d \omega)^{r} \equiv 0
$$

The Kupka set of the distribution $\mathcal{D}$ induced by $\omega \in H^{0}\left(M, \Omega_{M}^{1}(L)\right)$ is defined by

$$
K(\mathcal{D})=\left\{p \in M ; \omega(p)=0,(d \omega)^{r}(p) \neq 0\right\} \subset \operatorname{Sing}(\mathcal{D}) .
$$

Theorem 4. Let $\mathcal{D}$ be a codimension one distribution on $\mathbb{P}^{n}$ of class $r$, given by a 1 -form $\omega \in H^{0}\left(\mathbb{P}^{n}, \Omega_{\mathbb{P}_{n}}^{1}(d+2)\right)$ such that codSing $\left((d \omega)^{r}\right) \geq 3$. Suppose that the Kupka component of $\mathcal{F}$ has transversal type

$$
\sum_{i=0}^{r-1}\left(x_{i} d x_{i+r}-x_{i+r} d x_{i}\right) .
$$

Then $\mathcal{D}$ is induced, in homogenous coordinates, by the 1-form

$$
\sum_{i=0}^{r-1}\left(f_{i} d f_{i+r}-f_{i+r} d f_{i}\right)
$$

\section{The Kupka Set.}

In this section, we study the main properties of the Kupka set. First, we study the behavior on a neighborhood of the Kupka set. As we will see, the geometric properties of the Kupka set, implies a strong rigidity on the global behavior of the foliation. 


\subsection{Basic properties of the Kupka set.}

Definition 2.1. Let $\Omega \in H^{0}\left(M, \Omega^{n-k}(L)\right)$ be an integrable section. The Kupka singular set is defined by

$$
K(\mathcal{F})=K(\Omega):=\{p \in M \mid \Omega(p)=0, d \Omega(p) \neq 0\} .
$$

The Kupka set is well defined. Let $\Omega$ an integrable $(n-k)$ form andy let $\Omega^{\prime}$ be another integrable form which define the same foliation, then

$$
\Omega=h \cdot \Omega^{\prime}, \quad h \in \mathcal{O}^{*}
$$

then $\Omega(x)=h(x) \cdot \Omega^{\prime}(x)=0$ if and only if $\Omega(x)=\Omega^{\prime}(x)=0$, and for any $x \in S(\Omega)=S\left(\Omega^{\prime}\right)$ we have $d \Omega(x)=h(x) \cdot d \Omega^{\prime}(x)$. Therefore $d \Omega(x) \neq 0$ if and only if $d \Omega^{\prime}(x) \neq 0$.

Now, we begin with the local product structure.

Theorem 2.2 (Medeiros [20]). Let $\mathcal{F} \in \mathcal{F}_{k}(M, L)$ be a $k$-dimensional holomorphic foliation on $M$ and let $K \subset K(\mathcal{F})$ be a connected component. Then there exists a germ of a holomorphic vector field $\mathbf{X} \in \Theta_{0}\left(\mathbb{C}^{n-k+1}\right)$ called the transversal type and open covering by charts $\left\{\left(U_{\alpha}, \phi_{\alpha}\right)\right\}$ of a neighborhood of $K$ such that

(1) $K(\mathcal{F})$ is a $k-1$-dimensional submanifold of $M$. Namely

$$
\begin{aligned}
\phi_{\alpha}: U_{\alpha} & \rightarrow \mathbb{C}^{n-k+1} \times \mathbb{C}^{k-1} \\
u & \mapsto\left(x_{\alpha}(u), y_{\alpha}(u)\right), \quad U_{\alpha} \cap K=\phi_{\alpha}^{-1}\left(0, y_{\alpha}\right)
\end{aligned}
$$

(2) The $(n-k)$-form

$$
\Omega_{\alpha}=\phi_{\alpha}^{*}\left(\imath_{\mathbf{X}} d x_{0} \wedge \ldots \wedge d x_{n-k}\right) .
$$

represents the foliation on $U_{\alpha}$.

(3) $K(\mathcal{F})$ is persistent under variation of $\Omega$; namely, for $p \in K(\mathcal{F})$ with transversal type $\eta$ andy for any foliation $\mathcal{F}^{\prime}$ sufficiently close to $\mathcal{F}$, there is a holomorphic $p$-form $\eta^{\prime}$, close to $\eta$ and defined on a neighborhood of $0 \in \mathbb{C}^{n-k+1}$ and a submersion $\phi^{\prime}$ close to $\phi$, such that $\mathcal{F}^{\prime}$ is defined by $\left(\phi^{\prime}\right)^{*} \eta^{\prime}$ on a neighborhood of $p$.

Remark 2.3. Let us explain the point (3) of the Theorem (2.2) for the families $\mathcal{K}_{k}(M, L)$ on a compact complex manifold $M$.

Let $\left\{\mathcal{F}_{t}\right\}_{t \in \mathcal{T}}$ a family of foliations such that $\mathcal{F}_{0} \in \mathcal{K}_{k}(M, L)$. Then as a consequence of (3), we get a family of compact submanifolds $\jmath_{t}: K_{t} \hookrightarrow M$ such that $\jmath\left(K_{t}\right)=K\left(\mathcal{F}_{t}\right)$ and having transversal type $\mathbf{X}_{t}$.

The possible transversal type, is a germ at zero of holomorphic vector field, it belongs to a space of infinite dimension, but $\mathcal{K}_{k}(M, L)$ has finite dimension.

For instance, for the rational components $\mathcal{R}\left(n: d_{0}, \ldots, d_{n-k}\right)$ defined in (4.3), the transversal type remain fixed for any family $\mathcal{F}_{t} \in \mathcal{R}\left(n: d_{0}, \ldots, d_{n-k}\right)$

$$
\mathbf{X}_{t}=\sum_{i=0}^{n-k} d_{j} x_{j} \frac{\partial}{\partial x_{j}}
$$

Given a foliation $\mathcal{F}$ represented by a section $\Omega \in H^{0}\left(M, \Omega^{n-k}(L)\right)$. Recall that the stalk at $x \in M$ of the tangent sheaf $\left(T_{\mathcal{F}}\right)_{x} \subset \Theta_{x}$, consist of the sheaf of the germs of holomorphic vector fields that vanishes the form $\Omega$.

$$
\left(T_{\mathcal{F}}\right)_{x}=\left\{\mathbf{Y} \in \Theta_{x} \mid \imath_{\mathbf{Y}} \Omega=0\right\} .
$$

It follows that there is a coordinate system around each point of the Kupka set $(x, y) \in \mathbb{C}^{n-k+1} \times \mathbb{C}^{k-1} \simeq \mathbb{C}^{n}$, the form $(n-k)$-form

$$
\Omega=\Omega(x, y)=\imath_{\mathbf{X}} d x_{0} \wedge \cdots \wedge d x_{n-k}
$$


defines the foliation. Then, the holomorphic vector fields

$$
\left\{\mathbf{X}(x), \frac{\partial}{\partial y_{1}}, \ldots, \frac{\partial}{\partial y_{k-1}}\right\}
$$

generate the tangent sheaf of the foliation as a $\mathcal{O}$ module around $x \in K$, therefore, it is locally free.

2.2. Geometry of the Kupka set. We assume that $K \subset K(\mathcal{F})$ is a compact and connected component

We say that a compact (not necessarily connected) submanifold $X \hookrightarrow M$ is subcanonicaly embedded, if its canonical bundle extends to a line bundle defined on $M$. That is

$$
\mathcal{K}_{X}=\left.L\right|_{X} \quad \text { for some } \quad L \in \operatorname{Pic}(M)
$$

Let $\mathcal{F}$ be a foliation with a compact Kupka component $K$. Consider now the family of $(n-k)$-forms $\left\{\Omega_{\alpha}=\phi_{\alpha}^{*} \Omega\right\}$ as in the theorem (2.2). Since $d \Omega(p) \neq 0$ at $p \in K(\Omega)$, we have

$$
d \Omega_{\alpha}(p)=\phi_{\alpha}^{*} d \Omega\left(0, y_{\alpha}\right)=\operatorname{Div}(\mathbf{X})(0) d x_{\alpha}^{0} \wedge \cdots \wedge d x_{\alpha}^{n-k} \neq 0,
$$

where

$$
\operatorname{Div}(\mathbf{X})(0)=\sum_{i=0}^{n-k} \frac{\partial X_{i}}{\partial x_{i}}(0)
$$

Now, we are in a position to prove the following geometric property of the Kupka set.

Proposition 2.4. Let $\mathcal{F} \in \mathcal{K}_{k}(M, L)$ with a compact Kupka component $K(\mathcal{F})=K$. Let $\mathrm{\jmath}: K(\mathcal{F}) \hookrightarrow M$ be the inclusion map. Then

(1) $\jmath: K(\mathcal{F}) \hookrightarrow M$ is subcanonicaly embedded.

$$
\wedge^{n-k+1} \nu(K(\mathcal{F}), M)=\left.L\right|_{K(\mathcal{F})}, \quad \mathcal{K}_{K(\mathcal{F})}=\left.\left(\mathcal{K}_{M} \otimes L\right)\right|_{K(\mathcal{F})}
$$

(2) $c_{1}\left(\nu(K, M)=\jmath^{*} c_{1}\left(N_{\mathcal{F}}\right) \in H^{2}(K, \mathbb{Z})\right.$.

In particular, for a foliation on the projective space $\mathbb{P}^{n}, K$ is Fano if and only if $\operatorname{dim}(\mathcal{F})>\operatorname{deg}(\mathcal{F})$.

Proof. Denote by $K=K(\mathcal{F})$. The cocycle condition $\Omega_{\alpha}=\lambda_{\alpha \beta} \Omega_{\beta}$, implies

$$
d \Omega_{\alpha}=d \lambda_{\alpha \beta} \wedge \Omega_{\beta}+\lambda_{\alpha \beta} d \Omega_{\beta} .
$$

Therefore, $d \Omega_{\alpha}=\lambda_{\alpha \beta} d \Omega_{\beta}$ in $K \cap U_{\alpha \beta}$. It follows that $\left\{\left.d \Omega_{\alpha}\right|_{K}\right\}$ defines a never vanishing holomorphic section of the line bundle

$$
\left.\wedge^{n-k+1} \nu(K, M)^{*} \otimes L\right|_{K}
$$

Hence, this line bundle is trivial. This implies that

$$
\left.\wedge^{n-k+1} \nu(K, M) \simeq L\right|_{K}=\left.\operatorname{det}\left(N_{\mathcal{F}}\right)\right|_{K}
$$

From the exact sequence of vector bundles over the Kupka set

$$
\left.\mathbf{0} \rightarrow T K \rightarrow T M\right|_{K} \rightarrow \nu(K, M) \rightarrow \mathbf{0},
$$

after taking the dual sequence and the exterior power we get

$$
\left.\mathcal{K}_{M}\right|_{K}=\wedge^{n-k+1} \nu(K, M)^{*} \otimes \mathcal{K}_{K} \Rightarrow \mathcal{K}_{K}=\left.\left(\mathcal{K}_{M} \otimes L\right)\right|_{K}
$$

It follows from the definition of the first Chern class:

$$
c_{1}(\nu(K, M))=c_{1}\left(\wedge^{n-k+1} \nu(K, M)\right)=\jmath^{*} c_{1}(L)=\jmath^{*} c_{1}\left(N_{\mathcal{F}}\right) .
$$

We proceed with another proof of the first item of the theorem above: 
Proof. We first observe that $\left.\left(T_{\mathcal{F}}\right)\right|_{K}=T K$. In fact, on the coordinate system $(U, \phi)$ given by Theorem (2.2), the tangent sheaf of the foliation, is generated by the vectors fields

$$
\begin{aligned}
T_{\mathcal{F}}(U) & =\mathcal{O}_{M}(U) \cdot\left\langle\frac{\partial}{\partial y_{1}}, \ldots, \frac{\partial}{\partial y_{k-1}}, \mathbf{X}(x)\right\rangle \\
\left.T K\right|_{U} & =\mathcal{O}_{K} \cdot\left\langle\frac{\partial}{\partial y_{1}}, \ldots, \frac{\partial}{\partial y_{k-1}}\right\rangle
\end{aligned}
$$

Now, we have that $\mathbf{X}(0) \equiv 0$, this implies that

$$
\mathcal{O}_{K} \cdot T_{\mathcal{F}}=\mathcal{O}_{K} \cdot\left\langle\frac{\partial}{\partial y_{1}}, \ldots, \frac{\partial}{\partial y_{k-1}}, \mathbf{X}(0)\right\rangle=\mathcal{O}_{K} \cdot\left\langle\frac{\partial}{\partial y_{1}}, \ldots, \frac{\partial}{\partial y_{k-1}}\right\rangle
$$

Therefore, the canonical bundle of the Kupka set is the restriction of the canonical bundle of the foliation, that means

$$
\mathcal{K}_{K}=\left.\left(\mathcal{K}_{\mathcal{F}}\right)\right|_{K(\mathcal{F})}=\left.\left(L \otimes \mathcal{K}_{M}\right)\right|_{K}, \quad \text { and } \quad \wedge^{n-k+1} \nu(K, M)=\left.L\right|_{K} .
$$

The second part follows in the same way.

Also, in the same coordinate system $(U, \phi)$, the normal bundle of the Kupka set is generated in $V=U \cap K$ by

$$
\left.\nu(K, M)\right|_{V}=\mathcal{O}_{K}(V) \cdot\left\langle\frac{\partial}{\partial x_{0}}, \ldots, \frac{\partial}{\partial x_{n-k}}\right\rangle \simeq \mathcal{O}_{K}^{n-k+1}(V),
$$

The normal sheaf, is coherent and it is not locally free at the singular set. It has the same generators, but it has the relation given by the transversal vector field $\mathbf{X}$, because it is tangent to the foliation. That is

$$
N_{\mathcal{F}}(U)=\frac{\mathcal{O}_{M}(U) \cdot\left\langle\frac{\partial}{\partial x_{0}}, \ldots, \frac{\partial}{\partial x_{n-k}}\right\rangle}{\sum X_{i}(x) \frac{\partial}{\partial x_{i}}}
$$

explicitly, we have the exact sequence

$$
\mathcal{O}_{M}(U) \stackrel{\cdot \mathbf{X}}{\longrightarrow} \mathcal{O}_{M}^{n-k+1}(U) \longrightarrow N_{\mathcal{F}}(U)
$$

Example 2.5. Here we have some examples and applications of the above result.

(1) The Proposition (2.4) andy the Gherardelli theorem, that states that any subcanonical and projectively normal curve of $\mathbb{P}^{3}$ is a complete intersection, we have that $\Gamma: \mathbb{P}^{1} \rightarrow \mathbb{P}^{3}$, the rational normal curve of degree 3 , parameterized by

$$
\Gamma(s: t)=\left(s^{3}: s^{2} t: s t^{2}: t^{3}\right)
$$

can't be the Kupka set of any codimension one foliation on $\mathbb{P}^{3}$.

(2) Any projective space linearly embedded, appears as a Kupka set of a degree zero foliation. Namely the foliation are the fibers of a linear projection $\mathbb{P}^{n} \rightarrow \mathbb{P}^{n-k}$ andy the Kupka set is the set of indetermination points.

On the other hand, any projective space $\mathbb{P}^{k-1}$ can not be the Kupka set of a foliation $\mathcal{F}$ of dimension $k$ and degree $\operatorname{deg}(\mathcal{F}) \geq k$.

2.3. The normal bundle of the Kupka set. Now, We to show another relevant property of the normal bundle of a Kupka component.

Let $\mathbf{X}=X_{1}+\ldots$ be the transversal vector field of a compact Kupka component. The linear part $X_{1}(x) \neq 0$. After a linear change of coordinates, we may assume 
that

$$
X_{1}(x)=\left(\begin{array}{cccc}
J_{\lambda_{1}} & 0 & \cdots & 0 \\
0 & J_{\lambda_{2}} & \cdots & 0 \\
\vdots & \ddots & \ddots & \vdots \\
0 & 0 & \cdots & J_{\lambda_{r}}
\end{array}\right) \cdot\left(\begin{array}{c}
x_{1} \\
x_{2} \\
\vdots \\
x_{r}
\end{array}\right) \quad x_{j} \in \mathbb{C}^{n_{j}}, \quad \sum_{j=1}^{r} n_{j}=n-k+1
$$

We assume that $J_{\lambda_{j}}$ are the Jordan blocs with $\lambda_{i} \neq \lambda_{j}$, but eventually, $J_{\lambda_{i}}$ could have several Jordan subblocs.

The Jordan canonical form, induces a decomposition in direct sum of invariant subspaces

$$
\mathbb{C}^{n-k+1}=\bigoplus_{i=1}^{r} V\left(\lambda_{i}\right), \quad \operatorname{dim}\left(V_{\lambda_{i}}\right)=n_{i} .
$$

Theorem 2.6. Let $\mathcal{F} \in \mathcal{K}_{k}(M, L)$ with transversal type $\mathbf{X}=X_{1}+\cdots$, assume that the linear part $X_{1}$ is in Jordan canonical form as in 2.1. Then the normal bundle decomposes as a direct sum of the type

$$
\nu(K, M)=\bigoplus_{i=1}^{r} E\left(\lambda_{i}\right) \text {, with } r k\left(E_{\lambda_{i}}\right)=n_{i}
$$

or it is indecomposable and projectively flat.

Proof. Let $\left(U_{\alpha}, \phi_{\alpha}\right)$ be the charts of the Theorem (2.2). The changes of coordinates $\phi_{\alpha \beta}:=\phi_{\beta} \circ \phi_{\alpha}$ satisfy $\phi_{\alpha \beta}^{*} \eta=\lambda \eta$ for some $\lambda \in \mathcal{O}^{*}$ andy $\eta=\imath_{\mathbf{X}} d x_{0} \wedge \cdots \wedge d x_{n-k}$.

Put in coordinates $\phi_{\alpha \beta}\left(x_{\beta}, y_{\beta}\right)=\left(x_{\alpha}, y_{\alpha}\right)$, then for the linear part, we have

$$
\left(\frac{\partial \phi_{\alpha \beta}}{\partial x_{\beta}}\right)_{*} X_{1}=\left(\frac{\partial \phi_{\alpha \beta}}{\partial x_{\beta}}\right) \cdot X_{1} \cdot\left(\frac{\partial \phi_{\alpha \beta}}{\partial x_{\beta}}\right)^{-1}=a X_{1} \quad a \in \mathbb{C}^{*} .
$$

Then, the change of coordinates of the normal bundle preserve the Jordan decomposition of the linear part.

Since a Jordan bloc has always an eigenvalue, the collection of these eigenvalues provide a subbundle.

If the linear part is diagonal with only one eigenvalue, has not a decomposition as a direct sum, but this case is projectively flat.

Remark 2.7. The normal bundle

$$
\nu(K, M)=\bigoplus_{i=1}^{r} E_{\lambda_{i}} \Rightarrow c\left(\nu(K, M)=\sum c_{1}\left(E_{\lambda_{i}}\right)\right.
$$

It would be interesting find relations between the Chern classes of the vector bundles $E_{\lambda_{i}}$, we conjecture that

$$
\lambda_{j} n_{i} c_{1}\left(E_{\lambda_{i}}\right)=\lambda_{i} n_{j} c_{1}\left(E_{\lambda_{j}}\right) \quad n_{i}=\operatorname{rank}\left(E\left(\lambda_{i}\right)\right),
$$

moreover, if $c_{1}\left(N_{\mathcal{F}}\right) \neq 0$ then $J_{\lambda_{i}}=\lambda_{i} \mathbb{I}_{n_{i} \times n_{i}}$.

This holds for foliations of codimension 1 and 2 ([8]).

Example 2.8. Let $E \rightarrow M$ be an irreducible projectively flat vector bundle. Let $L$ be a sufficiently holomorphic, ample line bundle such that $E \otimes L$ has sufficiently many holomorphic sections.

Now, let $\sigma \in H^{0}(M, E \otimes L)$ be a section vanishing transversely along a submanifold $K$. We consider the section $\sigma$ as a meromorphic section on $\mathbb{P}(E \otimes L)$, the foliation is the pull-back of the flat connection form on $\mathbb{P}(E \otimes L)$. 
Now, let $\mathcal{F}$ be a foliation with a compact Kupka component $K$. Assume that the line bundle $L=\operatorname{det}\left(N_{\mathcal{F}}\right)$ is ample and transversal type of the Kupka component is the vector field

$$
\mathbf{X}\left(x_{0}, \ldots, x_{n-k}\right)=\sum_{i=0}^{n-k}\left(\lambda_{i} x_{i}+\cdots\right) \frac{\partial}{\partial x_{i}} \quad \lambda_{i}-\lambda_{j} \neq 0 \neq \lambda_{i} \text { for all } i \neq j,
$$

in this case, we also have that $\operatorname{Div}(\mathbf{X})_{0}=\sum \lambda_{i} \neq 0$.

In this situation, it is shown in [8], that the eigendirections of the transversal vector field, defines holomorphic line bundles $L_{i}$ over $K$ such that

$$
\nu\left(K, \mathbb{P}^{n}\right)=\bigoplus_{i=0}^{n-k} L_{i} \Longrightarrow c_{1}\left(\nu\left(K, \mathbb{P}^{n}\right)\right)=\sum c_{1}\left(L_{i}\right)=\jmath^{*}\left(c_{1}\left(N_{\mathcal{F}}\right)\right) \neq 0 .
$$

On the other hand, the Chern classes $c_{1}\left(L_{j}\right) \in H^{2}(K, \mathbb{Z}) \quad j=0, \ldots n-k$ satisfy the following relations.

$$
\lambda_{j} \cdot c_{1}\left(L_{i}\right)-\lambda_{i} \cdot c_{1}\left(L_{j}\right)=0 \in H^{2}(K, \mathbb{Z}), \quad \forall i, j=0, \ldots n-k,
$$

it follows that the eigenvalues $\left(\lambda_{0}, \ldots, \lambda_{n-k}\right)$ of the linear part of the transversal type $\mathbf{X}$ may be taken to be integers

A combination of the relations (2.2) and (2.3) gives us that the Chern classes of the line bundles $L_{i}$ are restriction of classes on the ambient manifold. Namely

and then, we have

$$
\jmath^{*}\left(c_{1}\left(N_{\mathcal{F}}\right)\right)=\sum_{i=0}^{n-k} c_{1}\left(L_{i}\right)=\left[\sum_{i=0}^{n-k}\left(\frac{\lambda_{i}}{\lambda_{j}}\right)\right] \cdot c_{1}\left(L_{j}\right),
$$

$$
H^{2}(K, \mathbb{Z}) \ni c\left(L_{i}\right)=\frac{\lambda_{i}}{\sum_{i=0}^{n-k} \lambda_{i}} \jmath^{*}\left(c_{1}\left(N_{\mathcal{F}}\right)\right)=\jmath^{*}\left(\frac{\lambda_{i} c_{1}\left(N_{\mathcal{F}}\right)}{\sum_{i=0}^{n-k} \lambda_{i}}\right)
$$

We observe that the right side of the above equation, belongs to $H^{2}(M, \mathbb{Q})$.

Now, assume that the line bundles $L_{i}$ are restriction of holomorphic line bundles $\overline{L_{i}} \in \operatorname{Pic}(M)$.

Then we, get a collection of $(n-k+1)$ integer classes

such that,

$$
c_{i}=\frac{\lambda_{i} c_{1}\left(N_{\mathcal{F}}\right)}{\sum_{i=0}^{n-k} \lambda_{i}} \in H^{2}(M, \mathbb{Z}) \quad i=0, \ldots, n-k
$$

$$
c_{0}+\cdots+c_{n-k}=c_{1}\left(N_{\mathcal{F}}\right)
$$

We will find conditions for foliations on the projective space, such that the line bundles on the Kupka set are restrictions of line bundles on the projective space.

We give now other examples of submanifolds that can not be the Kupka set of any foliation.

Example 2.9. Let $\mathcal{S}_{p, q}: \mathbb{P}^{p} \times \mathbb{P}^{q} \hookrightarrow \mathbb{P}^{(p+1)(q+1)-1}$ be the Segre embedding. We will see that for any $c \in \mathbb{Z}$, the image $\mathcal{S}_{d-1,1}\left(\mathbb{P}^{d-1} \times \mathbb{P}^{1}\right)=\Sigma_{d} \subset \mathbb{P}^{2 d-1}$ cannot be the Kupka set of any foliation $\mathcal{F} \in \mathcal{F}_{d+1}(2 d-1, c)$.

We use the fact that the normal bundle $\nu\left(\Sigma_{d}, \mathbb{P}^{2 d-1}\right)$ is irreducible 11. Then, by the Theorem (2.6), the normal bundle is projectively flat and the linear transversal type must be radial. By the Poincaré linearization theorem, the transversal type is actually radial.

The normal bundle $\nu\left(\Sigma_{d}, \mathbb{P}^{2 d-1}\right)$ is projectively flat. Then the bundle

$$
E=\nu\left(\Sigma_{d}, \mathbb{P}^{2 d-1}\right) \otimes \mathcal{O}_{\Sigma_{d}}(-c / d-1) \text { has } c(E)=1 \in H^{*}\left(\Sigma_{d}, \mathbb{Z}\right)
$$


and by [18, pages $114-115]$, it is flat. Since $\Sigma_{d}$ is simply connected, the bundle $E$ is trivial. Therefore

$$
\nu\left(\Sigma_{d}, \mathbb{P}^{2 d-1}\right)=E \otimes \mathcal{O}_{\Sigma_{d}}(c /(d-1))=\bigoplus^{d-1} \mathcal{O}_{\Sigma_{d}}(c /(d-1))
$$

In the codimension one case, i. e. $\mathbb{P}^{2} \times \mathbb{P}^{1} \simeq \Sigma_{3} \subset \mathbb{P}^{5}$, we have a direct proof without the Theorem (2.6).

We will prove that, if $\Sigma_{3} \subset \mathbb{P}^{5}$ is the Kupka set of a codimension one holomorphic foliation, then its normal bundle $\nu\left(\Sigma_{3}, \mathbb{P}^{5}\right) \simeq L_{0} \oplus L_{1}$ for some holomorphic vector bundles of rank one.

Recall that for codimension one foliations (see for instance [6]), the transversal type is given by a linearizable and diagonal vector field

$$
\mathbf{X}\left(x_{0}, x_{1}\right)=\lambda_{0} x_{0} \frac{\partial}{\partial x_{0}}+\lambda_{1} x_{1} \frac{\partial}{\partial x_{1}},
$$

where the eigenvalues are positive integers.

We have two cases:

First, if $\lambda_{0} \neq \lambda_{1}$ then $\nu\left(\Sigma_{3}, \mathbb{P}^{5}\right)=L_{0} \oplus L_{1}$ and the normal bundle splits.

If $\lambda_{0}=\lambda_{1}=1$. In this case, we have that $c_{1}\left(N_{\mathcal{F}}\right)=c$ is even, since the degree of the Kupka set is $\operatorname{deg}(K)=c^{2} / 4$ (see 3.3) and $\nu\left(\Sigma_{3}, \mathbb{P}^{5}\right)$ is projectively flat. Therefore the vector bundle

$$
E=\nu\left(\Sigma_{3}, \mathbb{P}^{5}\right) \otimes \mathcal{O}_{\Sigma_{3}}(-c / 2)
$$

has $c_{1}(E)=c_{2}(E)=0$. It follows from [18] pag. 114-115, that it is flat.

Since $\Sigma_{3}$ is simply connected, then $E=\mathcal{O}_{\Sigma_{3}} \oplus \mathcal{O}_{\Sigma_{3}}$. Then

$$
\nu\left(\Sigma_{3}, \mathbb{P}^{5}\right)=E \otimes \mathcal{O}_{\Sigma_{3}}(c / 2)=\mathcal{O}_{\Sigma_{3}}(c / 2) \oplus \mathcal{O}_{\Sigma_{3}}(c / 2) .
$$

Again in this case the normal bundle splits.

The properties of the normal bundle imposses many restrictions on the Kupka set.

We use the following result [3].

Theorem 2.10. Let $X \subset \mathbb{P}^{4}$ be a smooth surface. If the normal bundle is decomposable i.e. $\nu\left(X, \mathbb{P}^{4}\right)=L_{1} \oplus L_{2}$ for some $L_{1}, L_{2} \in \operatorname{Pic}(X)$, then $X$ is a complete intersection.

With this result, we are able to prove:

Theorem 2.11. Set $\mathcal{F} \in \mathcal{K}_{3}(4, c)$. If the transversal type is not the radial vector field or it is radial but $K$ is simply connected, then $K$ is a complete intersection and $\mathcal{F}$ has a meromorphic first integral.

Proof. The transversal type is $\mathbf{X}_{p q}$ for some integers $1 \leq p<q$ or $p=q=1$. In the first case, the normal bundle splits in a sum of line bundles.

If $p=q=1$, then $\nu\left(K, \mathbb{P}^{4}\right)$ is projectively flat and the foliation has even first Chern class andy $\nu\left(K, \mathbb{P}^{4}\right)(-c / 2)$ is flat. Since $\pi_{1}(K, *)=1$, it is trivial and $\nu\left(K, \mathbb{P}^{4}\right)=\mathcal{O}_{K}(c / 2) \oplus \mathcal{O}_{K}(c / 2)$. Anyway, the conclusion follows from (2.10).

Recall that in the codimension one case, for a compact, conected component of the Kupka set, there exists a rank two holomorphic vector bundle $E$, with a section $\sigma$, and the exact sequence

$$
0 \rightarrow \mathcal{O} \stackrel{\sigma}{\rightarrow} E \rightarrow \mathcal{J}_{K}(c) \rightarrow 0 .
$$

The Kupka set is a complete intersection if and only if $E$ splits [9].

As a consequence of the Horrocks criteria for splitting of holomorphic vector bundles on the projective space, we have 
Corollary 2.12. Set $\mathcal{F} \in \mathcal{K}_{n-1}(n, c) \quad n \geq 5$, then $K$ is a complete intersection.

Proof. Let $\mathcal{F} \in \mathcal{K}_{4}(5, c)$ with Kupka set $K$. Then $K$ is simply connected and by Lefschetz, it hyperplane section also is a simply connected. Let $\ell: \mathbb{P}^{4} \rightarrow \mathbb{P}^{5}$ a linear embedding and $\ell^{*} \mathcal{F}$ has a complete intersection Kupka set $\ell^{-1} K$. Hence $K$ is a complete intersection.

Example 2.13. It is shown in [9, that the complex Torus $\mathbb{C}^{2} / \Lambda \subset \mathbb{P}^{4}$, associated to the Horrocks-Munford bundle, can not be the Kupka set for a foliations on $\mathbb{P}^{4}$.

The Horrocks-Munford bundle is stable, but this property never holds for the rank two holomorphic vector bundle associated to a Kupka component.

2.4. Normal Forms. Through this subsection, we assume that the transversal type of a compact Kupka component is a linear vector field of the type

$$
\mathbf{X}=\sum_{i=0}^{n-k} \lambda_{i} z_{i} \frac{\partial}{\partial z_{i}}, \quad \lambda_{i} \neq 0 \neq \lambda_{i}-\lambda_{j}, \quad i, j=0 \ldots n-k
$$

and $\Omega=\imath_{\mathbf{X}} d z_{0} \wedge \cdots \wedge d z_{n-k}$.

We consider such a vector field, as a vector in $\Lambda \in \mathbb{C}^{n+k+1}$, where $\Lambda=\left(\lambda_{0}, \ldots, \lambda_{n-k}\right)$.

Definition 2.14. $\Lambda=\left(\lambda_{0}, \ldots, \lambda_{n-k}\right)$ is resonant if among the eigenvalues there exists integer relations of the type

$$
\lambda_{s}=\langle\mathbf{m}, \Lambda\rangle=\sum_{j=0}^{n-k} m_{j} \lambda_{j}
$$

where $\mathbf{m}=\left(m_{0}, \ldots, m_{n-k}\right), \quad m_{j} \geq 0, \quad \sum m_{j} \geq 2$. Such a relation is called a resonance. The number $|\mathbf{m}|=\sum m_{j}$ is called the order of the resonance.

Resonances are the obstructions for formal linearization of vector fields of the type $\mathbf{Y}=\mathbf{X}+\cdots$. For us, an interpretation of the meaning of the resonance is the following.

For a linear vector field $\mathbf{X}$, for all $j=0, \ldots n-k$, the hyperplanes $\left\{z_{j}=0\right\}$ are keeping invariant. If there is a resonance of the type $\lambda_{k}=\langle\mathbf{m}, \Lambda\rangle$ the families of hypersurfaces $z_{k}+c z_{0}^{m_{0}} \cdots z_{n-k}^{m_{n-k}}=z_{k}+c Z^{\mathbf{m}}$ are also invariants by $\mathbf{X}$. Namely

$$
\mathbf{X}\left(z_{k}+c Z^{\mathbf{m}}\right)=\lambda_{k} z_{k}+c \mathbf{X}\left(Z^{\mathbf{m}}\right)=\lambda_{k} z_{k}+c \sum m_{j} \lambda_{j} Z^{m}=\lambda_{k}\left(z_{k}+c Z^{\mathbf{m}}\right) .
$$

Observe that the smooth hypersurfaces $\left\{\left(z_{k}+c Z^{\mathbf{m}}\right)=0\right\}$ and $\left\{z_{k}=0\right\}$ are tangent at the origin.

The vector polynomials $Z^{\mathbf{m}} \mathbf{e}_{j}$ are resonant if there is a resonance of the type $\lambda_{j}=\langle\mathbf{m}, \Lambda\rangle$. It follows that

$$
\mathbf{X}\left(Z^{\mathbf{m}} \mathbf{e}_{j}\right)=\left[\langle\mathbf{m}, \Lambda\rangle-\lambda_{j}\right] Z^{\mathbf{m}} \mathbf{e}_{j}
$$

In the sequel, we consider the forms $\Omega=\imath_{\mathbf{X}} d z_{0} \wedge \cdots \wedge d z_{n-k}$ with the assumption that $\left(\lambda_{0}, \ldots, \lambda_{n-k}\right)$ are positive integers.

We divide our analysis in three cases. The non-resonant, the resonant and the radial as transversal type of a Kupka component.

2.4.1. Non-resonant case.

Theorem 2.15. Let $K$ be a compact connected component of the Kupka set with transversal type

$$
\mathbf{X}=\sum_{i=0}^{n-k} \lambda_{i} z_{i} \frac{\partial}{\partial z_{i}} \quad \text { where } \quad \lambda_{i} \in \mathbb{N}
$$

and no-resonant. Then there exists a neighborhood $K \subset U$ such that $\left.\mathcal{F}\right|_{U}$ is defined by a meromorphic closed $(n-k)$ form with poles along an invariant divisor. 
Proof. Consider $\left(U_{\alpha}, \phi_{\alpha}\right)$ a covering by charts of the Kupka set andy $\Omega_{\alpha}=\phi_{\alpha}^{*} \Omega$.

In this case, we have that for all $j=0, \ldots, n-k$, the hyperplanes $\left\{z_{j}=0\right\}$ are the only smooth invariant hypersurfaces, then we get

$$
\Omega_{\alpha}=\sum_{j=0}^{n-k} \lambda_{j} z_{\alpha}^{j} d z_{\alpha}^{j+1} \wedge \cdots \wedge d z_{\alpha}^{j-1}
$$

moreover, for all $j=0, \ldots, n-k$, we have $z_{\alpha}^{j}=g_{\alpha \beta}^{j} z_{\beta}^{j}$, for some $g_{\alpha \beta}^{j} \in \mathcal{O}^{*}\left(U_{\alpha \beta}\right)$.

Consider the meromorphic closed forms

$$
\eta_{\alpha}=\frac{\Omega_{\alpha}}{z_{\alpha}^{0} \cdots z_{\alpha}^{n-k}}=\frac{\lambda_{\alpha \beta}}{g_{\alpha \beta}^{0} \cdots g_{\alpha \beta}^{n-k}} \frac{\Omega_{\beta}}{z_{\beta}^{0} \cdots z_{\beta}^{n-k}}=\frac{\lambda_{\alpha \beta}}{g_{\alpha \beta}^{0} \cdots g_{\alpha \beta}^{n-k}} \eta_{\beta} .
$$

We have the following cocycles

$$
c_{\alpha \beta}=\frac{\lambda_{\alpha \beta}}{g_{\alpha \beta}^{0} \cdots g_{\alpha \beta}^{n-k}} \in \mathcal{O}^{*}\left(U_{\alpha \beta}\right) .
$$

We claim that $c_{\alpha \beta} \equiv 1$. In fact, since $\eta_{\alpha}$ and $\eta_{\beta}$ are closed andy $\eta_{\alpha}=c_{\alpha \beta} \eta_{\beta}$, after taking exterior derivative we have $0=d c_{\alpha \beta} \wedge \eta_{\beta}$. It follows that $c_{\alpha \beta}$ is a holomorphic first integral of the vector field $\mathbf{X}$, since the eigenvalues are positive, any solution of the form accumulate at 0 , therefore $c_{\alpha \beta}$ is a constant.

On the other hand, the forms $\eta_{\alpha}$ and $\eta_{\beta}$ have the same poles, it follows that the constant $c_{\alpha \beta}=1$.

Therefore, $\eta_{\alpha}=\eta_{\beta}$ andy then, there exists a meromorphic closed $(n-k)$ form $\eta$ defined in $U=\cup U_{\alpha} \supset K$, with poles on an invariant divisor, and representing the foliation. This proof the non-resonant case.

2.4.2. Resonant Case. Again, consider the diagonal vector field

$$
\mathbf{X}=\sum_{i=0}^{n-k} \lambda_{i} z_{i} \frac{\partial}{\partial z_{i}}, \quad \lambda_{i} \in \mathbb{N}
$$

and the $n-k$ form

$$
\Omega=\sum_{j=0}^{n-k}(-1)^{j} \lambda_{j} x_{j} d x_{0} \wedge \cdots \wedge \widehat{d x_{j}} \wedge \cdots \wedge d x_{n-k} .
$$

Now, assume that the subset of eigenvalues $\Lambda_{N R}=\left\{\lambda_{0}<\lambda_{1}<\cdots<\lambda_{\ell}\right\}$ is non-resonant and maximal with this property. It is always non empty $\lambda_{0} \in \Lambda_{N R}$. The set $\Lambda_{R}=\left\{\lambda_{\ell+1}<\cdots<\lambda_{n-k}\right\}$ have resonances, that always may be found of the type

$$
\lambda_{\ell+j}=\lambda_{0} m_{0}^{\ell+j}+\cdots+\lambda_{\ell} m_{\ell}^{\ell+j}, \quad|\mathbf{m}| \geq 2
$$

involving only non-resonant eigenvalues.

In order to see that, we observe first that $\lambda_{\ell+1}$ is the smaller of all resonant eigenvalues andy it has an expression involving only non-resonant eigenvalues.

We proceed by induction over $k$, with the index $\ell+k$ andy prove for $\ell+k+1$. Assume that the statement is true for $\ell+k$ andy now, we have

$$
\lambda_{\ell+k+1}=\sum_{j=0}^{s} m_{j}^{k+1} \lambda_{j}
$$

since it involves only terms $\lambda_{j}<\lambda_{\ell+k+1}$. In particular, in this sum only could appear resonant eigenvalues $\lambda_{\ell+t}$ for $1 \leq t \leq k$. Then, these eigenvalues may be replaced by the non-resonant terms. Now, for any $s=1, \ldots n-k-\ell$, consider all elements

$$
R(s)=\left\{\mathbf{m} \in \mathbb{N}^{\ell} \mid\left\langle\mathbf{m}, \Lambda_{N R}\right\rangle=\lambda_{\ell+s}\right\}
$$


And for any $\mathbf{m}=\left(m_{0}, \ldots, m_{\ell}\right) \in R(s)$, we consider the rational functions

$$
\varphi_{\mathbf{m}}^{\ell+s}=\frac{x_{\ell+s}}{x_{0}^{m_{0}} \ldots x_{\ell}^{m_{\ell}}}=\frac{x_{\ell+s}}{x^{\mathbf{m}^{s}}} \quad \lambda_{\ell+s}=m_{0}^{s} \lambda_{0}+\cdots+m_{\ell}^{s} \lambda_{\ell}=\left\langle\mathbf{m}^{s}, \Lambda_{N R}\right\rangle .
$$

And for any $\mathbf{m}=\left(m_{0}, \ldots, m_{\ell}\right) \in R(s)$, we consider the rational functions

$$
\varphi_{\mathbf{m}}^{\ell+s}=\frac{x_{\ell+s}}{x_{0}^{m_{0}} \ldots x_{\ell}^{m_{\ell}}}=\frac{x_{\ell+s}}{x^{\mathbf{m}^{s}}} \quad \lambda_{\ell+s}=m_{0}^{s} \lambda_{0}+\cdots+m_{\ell}^{s} \lambda_{\ell}=\left\langle\mathbf{m}^{s}, \Lambda_{N R}\right\rangle .
$$

We will use the short notation. Define the monomial

$$
h_{\mathbf{m}}^{\ell+s}\left(x_{0}, \ldots, x_{\ell}\right)=x_{0}^{m_{0}^{s}} \ldots x_{\ell}^{m_{\ell}^{s}}, \quad \mathbf{m}=\left(m_{0}, \ldots, m_{\ell}\right) \in R(s),
$$

then we have

$$
x_{\ell+s}=h_{\mathbf{m}}^{\ell+s} \cdot \varphi_{\mathbf{m}}^{\ell+s}, \quad d x_{\ell+s}=\varphi_{\mathbf{m}}^{s} \cdot d h_{\mathbf{m}}^{s}+h_{\mathbf{m}}^{\ell+s} \cdot d \varphi_{\mathbf{m}}^{\ell+s}
$$

The function $h_{\mathbf{m}}^{\ell+s}$ is a resonant monomial associated to the eigenvalue $\lambda_{\ell+s}$ then we have, for instance $s=n-k-\ell$ that

$$
\begin{aligned}
\Omega & =\sum_{j=0}^{n-k}(-1)^{i} \lambda_{i} x_{i} d x_{0} \wedge \cdots \wedge \widehat{d x}_{i} \wedge \cdots \wedge d x_{n-k} \\
& =\varphi_{\mathbf{m}}^{n-k} \sum_{j=0}^{n-k-1}(-1)^{i} \lambda_{i} x_{i} d x_{0} \wedge \ldots \widehat{d x}_{i} \cdots \wedge d h_{\mathbf{m}}^{n-k} \\
& +(-1)^{n-k} \lambda_{n-k} x_{n-k} d x_{0} \wedge \cdots \wedge d x_{n-k-1} \\
& +h_{\mathbf{m}}^{n-k} \sum_{j=0}^{n-k-1}(-1)^{i} \lambda_{i} x_{i} d x_{0} \wedge \cdots \wedge \widehat{d x}_{i} \wedge \cdots \wedge d \varphi_{\mathbf{m}}^{n-k}
\end{aligned}
$$

Since $x_{n-k}=h_{\mathbf{m}}^{n-k} \varphi_{\mathbf{m}}^{n-k}$, and the resonance implies that

$$
\begin{aligned}
& \varphi_{\mathbf{m}}^{n-k} \sum_{j=0}^{n-k-1}(-1)^{i} \lambda_{i} x_{i} d x_{0} \wedge \ldots \widehat{d x_{i}} \cdots \wedge d x_{n-k-1} \wedge d h_{\mathbf{m}}^{n-k} \\
& +(-1)^{n-k} \lambda_{n-k}\left(\varphi_{\mathbf{m}}^{n-k} h_{\mathbf{m}}^{n-k}\right) d x_{0} \wedge \cdots \wedge d x_{n-k-1}=0 .
\end{aligned}
$$

We finally we get

$$
\Omega=h_{\mathbf{m}}^{k} \cdot\left(\sum_{j=0}^{n-k-1}(-1)^{i} x_{i} d x_{0} \wedge \ldots \widehat{d x}_{i} \ldots d x_{n-k-1} \wedge d \varphi_{\mathbf{m}}^{n-k}\right)
$$

This expression may be written in the form

$$
\Omega=h_{\mathbf{m}}^{n-k} \Omega_{1} \wedge d \varphi_{\mathbf{m}}^{n-k} \quad \Omega_{1}=\sum_{j=0}^{n-k-1}(-1)^{i} \lambda_{i} x_{i} d x_{0} \wedge \ldots \widehat{d x_{i}} \cdots \wedge d x_{n-k-1}
$$

By this procedure, we are able to get inductively the expression

$$
\begin{aligned}
\Omega & =h_{\mathbf{m}_{\ell+1}^{\ell+1}}^{\ell} \ldots h_{\mathbf{m}_{n-k}-k}^{n-1} \cdot\left(\Omega_{\ell} \wedge d \varphi_{\mathbf{m}_{\ell+1}^{\ell+1}}^{\ell} \cdots \wedge d \varphi_{\mathbf{m}_{n-k}}^{n-k}\right) \\
& =h_{\mathbf{m}_{\ell+1}^{\ell+1}} \ldots h_{\mathbf{m}_{n-k}}^{n-k} \cdot\left(\Omega_{\ell} \wedge d\left(\frac{x_{\ell+1}}{h_{\mathbf{m}_{\ell+1}^{\ell+1}}^{+1}}\right) \wedge \cdots \wedge d\left(\frac{x_{n-k}}{h_{\mathbf{m}_{n-k}-k}^{n-k}}\right)\right) .
\end{aligned}
$$

Now, we define the non-resonant part as the $\ell$-form

$$
\Omega_{N R}=\sum_{j=0}^{\ell}(-1)^{j} \lambda_{j} x_{j} d x_{0} \wedge \cdots \wedge \widehat{d x_{j}} \wedge \cdots \wedge d x_{\ell}
$$


and the logarithmic non-resonant part

$$
\eta_{N R}=\frac{\Omega_{N R}}{x_{0} \ldots x_{\ell}}=\sum_{j=0}^{\ell}(-1)^{j} \lambda_{j} \frac{d x_{0}}{x_{0}} \wedge \cdots \wedge \frac{\widehat{d x_{j}}}{x_{j}} \wedge \cdots \wedge \frac{d x_{\ell}}{x_{\ell}} .
$$

The above calculations implies the following theorem.

Theorem 2.16 (Local Resonant Normal Form). Consider

$$
\Omega=\sum_{j=0}^{n-k}(-1)^{i} \lambda_{i} x_{i} d x_{0} \wedge \ldots \widehat{d x_{i}} \cdots \wedge d x_{n-k}, \quad \lambda_{i} \in \mathbb{N}, \quad \lambda_{i} \neq \lambda_{j} \text { for } i \neq j
$$

Let $\Lambda_{N R}=\left\{\lambda_{0}<\cdots<\lambda_{\ell}\right\}$ and $\Lambda_{R}=\left\{\lambda_{\ell+1}<\cdots<\lambda_{n-k}\right\}$ be the non-resonant part and the resonant part respectively. Then there exists an integrating factor:

$$
\frac{\Omega}{x_{0} \ldots x_{\ell} H_{M}\left(x_{0}, \ldots x_{\ell}\right)}=\eta_{N R} \wedge d \varphi_{m_{\ell+1}^{\ell+1}} \wedge \cdots \wedge d \varphi_{m_{n-k}}^{n-k} .
$$

where $H_{\boldsymbol{M}}=h_{\boldsymbol{m}_{\ell+1}+1}^{++1} \ldots h_{\boldsymbol{m}_{n-k}}^{n-k}$, where $\boldsymbol{M}=\left(\boldsymbol{m}_{\ell+1}, \ldots, \boldsymbol{m}_{n-k}\right)$ and $\boldsymbol{m}_{\ell+s} \in R(s)$.

This expression gives an integrability condition with meromorphic closed forms. Observe that the function

$$
G_{\mathbf{M}}\left(x_{0}, \ldots, x_{\ell}\right)=x_{0} \ldots x_{\ell} H_{\mathbf{M}}\left(x_{0}, \ldots, x_{\ell}\right),
$$

is a holomorphic integrating factor of $\Omega$.

Also observe that if $\mathbf{m} \neq \mathbf{n} \in R(s)$, the differential of rational function $\varphi_{\mathbf{m}}^{\ell+s} / \varphi_{\mathbf{n}}^{\ell+s}$ divides the form $\Omega_{N R}$.

Now, we need to globalize this expression to a neighborhood of a Kupka set with transversal type $\Omega$.

We continue with the notation of the Theorem (2.16), but we fix (and omit) the subindexes $\mathbf{m} \in R(s)$ and $\mathbf{M}$, for the functions $h^{l+s}, H$, and $G$.

First, we observe that the pole of the logarithmic non-resonant part, is invariant by the foliation. Then, for all $j=0, \ldots, \ell, \quad\left\{x_{\alpha, j}=g_{\alpha \beta}^{j} x_{\beta, j}\right\}$. Also, we denote by $\zeta_{\alpha}=\left(x_{\alpha, 0}, \ldots, x_{\alpha, \ell}\right)$, then:

$$
h^{\ell+k}\left(\zeta_{\alpha}\right)=h_{\alpha}^{\ell+k} \quad H\left(\zeta_{\alpha}\right)=H_{\alpha} \quad G\left(\zeta_{\alpha}\right)=G_{\alpha}
$$

Set $\gamma_{\alpha \beta}=\left(g_{\alpha \beta}^{0}, \ldots, g_{\alpha \beta}^{\ell}\right)$. If we consider $\gamma_{\alpha \beta}$ as a diagonal matrix, then we have the equation $\zeta_{\alpha}=\gamma_{\alpha \beta} \zeta_{\beta}$. We also consider $\gamma_{\alpha \beta}$ as a vector andy we get the equations:

$$
h^{\ell+k}\left(\gamma_{\alpha \beta}\right):=h_{\alpha \beta}^{\ell+k} \quad H\left(\gamma_{\alpha \beta}\right):=H_{\alpha \beta} \quad G\left(\gamma_{\alpha \beta}\right):=G_{\alpha \beta} .
$$

and with this convention, we get the equations

$$
h_{\alpha}^{\ell+k}=h_{\alpha \beta}^{\ell+k} \cdot h_{\beta}^{\ell+k} \quad H_{\alpha}=H_{\alpha \beta} \cdot H_{\beta} \quad G_{\alpha}=G_{\alpha \beta} \cdot G_{\beta} .
$$

Thus, we have

$$
\frac{\Omega_{\alpha}}{G_{\alpha}}=\frac{\lambda_{\alpha \beta}}{G_{\alpha \beta}} \cdot \frac{\Omega_{\beta}}{G_{\beta}} .
$$

Since the forms are closed and in the Poincaré domain, it follows that the never vanishing holomorphic function

$$
\frac{\lambda_{\alpha \beta}}{G_{\alpha \beta}}=c_{\alpha \beta} \in \mathbb{C}^{*},
$$

but unfortunately not necessarily 1 . With the same notation, we can prove: 
Theorem 2.17. Let $K$ be a compact, connected Kupka component with transversal type $\Omega$ as described above. Then. There exists a neighborhood of the Kupka set such that the foliation is represented by a meromorphic $n-k$ form of the type.

$$
\eta=\eta_{N R} \wedge \theta_{\ell+1} \wedge \cdots \wedge \theta_{n-k}
$$

where $\theta_{j}, \quad j=1, \ldots, n-k-\ell$ are closed meromorphic 1 -forms.

Proof. The proof will be by induction on the number of ressonant eigenvalues.

Let $\mathcal{U}=\left\{U_{\alpha}\right\}$ a covering of the theorem (2.2) andy write $\Omega_{\alpha}$ in the local normal form.

$$
\Omega_{\alpha}=\eta_{\alpha} \wedge d \varphi_{\alpha}^{\ell+1} \wedge \cdots \wedge d \varphi_{\alpha}^{n-k}
$$

where $\eta_{\alpha}$ is logarithmic with poles on the divisor associated to the non-resonant eigenvalues.

Assume that $\Lambda_{N R}=\left\{\lambda_{0}, \ldots, \lambda_{n-k-1}\right\}$. Then for each $\alpha, \beta$ we have

$$
\eta_{\alpha} \wedge d \varphi_{\alpha}=c_{\alpha \beta} \eta_{\beta} \wedge d \varphi_{\beta} \quad c_{\alpha \beta} \in \mathbb{C}^{*} .
$$

Since $\eta_{\alpha}=\eta_{\beta}$, we get

$$
\eta_{\alpha} \wedge\left(d \varphi_{\alpha}-c_{\alpha \beta} d \varphi_{\beta}\right)=0 .
$$

The form $\eta_{\alpha}$ has the division property, then we get

$$
d \varphi_{\alpha}=c_{\alpha \beta} d \varphi_{\beta} \Rightarrow \varphi_{\alpha}=c_{\alpha \beta} \varphi_{\beta}+b_{\alpha \beta} \Rightarrow \frac{d \varphi_{\alpha}}{\varphi_{\alpha}}=\frac{d \varphi_{\beta}}{\varphi_{\beta}}
$$

Let $\theta_{\alpha}=d \log \varphi_{\alpha}$, we get a meromorphic form $\theta$ defined on the neighborhood $U=\bigcup_{\alpha} U_{\alpha}$ of $K$. We get

$$
\frac{\Omega}{G}=\eta_{N R} \wedge \theta
$$

Now, we assume that the result holds, if the number of resonant eigenvalues is $s$ and prove the theorem for $s+1$.

The local normal form is

$$
\eta_{\alpha} \wedge d \varphi_{\alpha}^{\ell+1} \wedge \cdots \wedge d \varphi_{\alpha}^{\ell+s+1} .
$$

with $\eta_{\alpha}=\eta_{\beta}$ the logarithmic part. The form

$$
\eta_{\alpha} \wedge d \varphi_{\alpha}^{\ell+1} \wedge \cdots \wedge d \varphi_{\alpha}^{\ell+s+1}=c_{\alpha \beta} \cdot \eta_{\beta} \wedge d \varphi_{\beta}^{\ell+1} \wedge \cdots \wedge d \varphi_{\beta}^{\ell+s+1} \quad c_{\alpha \beta} \in \mathbb{C}^{*} .
$$

By the induction hypotheses, we are able to write

$$
\eta_{\alpha} \wedge d \varphi_{\alpha}^{\ell+1} \wedge \cdots \wedge d \varphi_{\alpha}^{\ell+s}=a_{\alpha \beta} \cdot \eta_{\beta} \wedge d \varphi_{\beta}^{\ell+1} \wedge \cdots \wedge d \varphi_{\beta}^{\ell+s} \quad a_{\alpha \beta} \in \mathbb{C}^{*},
$$

we replaced by a form of the type

$$
\eta \wedge \theta_{\ell+1} \wedge \cdots \wedge \theta_{\ell+s} \quad \text { with } \quad d \theta_{j}=0 \quad \ell+1 \leq j \leq \ell+s .
$$

Then, we have a new meromorphic local model

$$
\omega_{\alpha}=\Theta \wedge d \varphi_{\alpha}^{\ell+s+1}
$$

where $\Theta=\eta \wedge \theta_{\ell+1} \wedge \cdots \wedge \theta_{\ell+s}$ and with $\eta$ a logarithmic $\ell$-form. Therefore

$$
\omega_{\alpha}=\Theta \wedge d \varphi_{\alpha}^{l+s+1}=a_{\alpha \beta}^{\prime} \cdot \Theta \wedge d \varphi_{\beta}^{l+s+1} \quad a_{\alpha \beta}^{\prime} \in \mathbb{C}^{*},
$$

As in the case of a single resonat eigenvalue, we have

$$
\Theta \wedge\left(d \varphi_{\alpha}^{\ell+s+1}-a_{\alpha \beta}^{\prime} \cdot d \varphi_{\beta}^{\ell+s+1}\right)=0 \Rightarrow \varphi_{\alpha}^{\ell+s+1}=a_{\alpha \beta}^{\prime} \varphi_{\beta}^{\ell+s+1}+b_{\alpha \beta} \quad b_{\alpha \beta} \in \mathbb{C} .
$$

Then $d \log \left(\varphi_{\alpha}^{\ell+s+1}\right)=d \log \left(\varphi_{\beta}^{\ell+s+1}\right)$ and we define the form

$$
\left.\left(\theta_{\ell+s+1}\right)\right|_{U_{\alpha}}=\frac{d \varphi_{\alpha}^{\ell+s+1}}{d \varphi_{\beta}^{\ell+s+1}}=\frac{d \varphi_{\beta}^{\ell+s+1}}{d \varphi_{\beta}^{\ell+s+1}}=\left.\left(\theta_{\ell+s+1}\right)\right|_{U_{\alpha}} .
$$

And finally, we get $\eta=\eta_{N R} \wedge \theta_{\ell+1} \wedge \cdots \wedge \theta_{\ell+s+1}$ with $d \theta_{j}=0$. 
As a conclusion, we have show the following result.

Theorem 2.18. Let $\mathcal{F}$ be a foliation with a Kupka component with transversal type

$$
\mathbf{X}=\sum_{j=0}^{n-k} \lambda_{i} z_{i} \frac{\partial}{\partial z_{i}}, \quad \lambda_{j} \neq \lambda_{i}, \quad \lambda_{i} \in \mathbb{N} .
$$

Then there exists a neighborhood $U$ of the Kupka set such that:

(1) There exists a meromorphic, closed and decomposable $n-k$ form defining $\mathcal{F}$ outside and invariant divisor

(2) The reduced invariant divisor $\mathcal{D}_{\text {red }}$ is a normal crossing divisor and it is the pole of the logarithmic non-resonant part.

Example 2.19. If the component has transversal type

$$
\mathbf{X}=\sum_{j=0}^{n-k} \lambda_{j} x_{j} \frac{\partial}{\partial x_{j}} \quad \lambda_{0}=1, \quad \lambda_{j}<\lambda_{j+1} \in \mathbb{N}
$$

then $\Lambda_{N R}=\left\{\lambda_{0}\right\}$ andy the forma may be written as

$$
\Omega=x_{0}^{N} d\left(\frac{x_{1}}{x_{0}^{\lambda_{1}}}\right) \wedge \cdots \wedge d\left(\frac{x_{n-k}}{x_{0}^{\lambda_{n-k}}}\right) \quad N=\sum_{j=0}^{n-k} \lambda_{j}
$$

2.4.3. The radial case. Now, we consider the case

$$
\mathbf{X}=\sum_{j=0}^{n-k} z_{i} \frac{\partial}{\partial z_{i}}
$$

Recall that a $(n-k)$ non singular foliation on a manifold $M$ has a transversal projective structure, if it is defined by an Atlas of submersions $f_{\alpha}: U_{\alpha} \rightarrow \mathbb{P}^{n-k}$ such that $f_{\alpha}=\phi_{\alpha \beta} \circ f_{\beta}$ and $\phi_{\alpha \beta}$ are restriction of projective transformations on $\mathbb{P}^{n-k}$.

The most simple example of transversally projective foliations, is given by fibrations $F: M \rightarrow \mathbb{P}^{n-k}$, another example are generated by suspensions of a representation $\pi_{1}(M) \rightarrow \mathbf{P G L}(n-k, \mathbb{C})$.

The more general transversally projective foliations are a mixture of these examples. It is given by the develop of the structure.

A singular holomorphic foliation has a projective transversal structure if outside the singular set, there exists an invariant divisor $D \subset M$ such that $\left.\mathcal{F}\right|_{M-D-S(\mathcal{F})}$ has a projective transversal structure, see [23].

Theorem 2.20. Let $K$ be a Kupka component with radial transversal type. Then there exists a neighborhood $K \subset U$ such that $\mathcal{F}$ has a projective transversal structure.

Proof. Let $K$ be a compact connected Kupka component of radial type andy let $\sigma: \widetilde{M}_{K} \rightarrow M$ be the blow-up along $K$. Denote by $E:=\sigma^{-1} K$ the exceptional divisor. It is well known that $E=\mathbb{P}(\nu(K, M))$, the projectivization of the normal bundle of $K \subset M$.

Consider now the charts $\left.\left\{U_{\alpha}, \phi_{\alpha}\right)\right\}$ of the theorem 2.2, with coordinates

$$
\phi_{\alpha}: U_{\alpha} \rightarrow \mathbb{C}^{n-k+1} \times \mathbb{C}^{k-1} \quad \phi_{\alpha}(p)=\left(x_{\alpha}^{0}, x_{\alpha}^{1}, \ldots, x_{\alpha}^{n-k}, y_{\alpha}^{1}, \ldots, y_{\alpha}^{k-1}\right)
$$

of the adapted covering of $K$. The $(n-k)$-form $\Omega_{\alpha}=\phi_{\alpha}^{*}(\eta)$, where

$$
\eta=\sum_{i=0}^{n-k}(-1)^{i} x_{i} d x^{0} \wedge \ldots \wedge \widehat{d x^{i}} \wedge \ldots \wedge d x^{n-k}
$$


as a vector field, the transversal type is

$$
\mathbf{X}=\sum_{i=0}^{n-k} x_{i} \frac{\partial}{\partial x_{i}}
$$

We consider the covering of the exceptional divisor

$$
U_{\alpha} \times \mathbb{P}^{n-k}=\left\{\left(x_{\alpha}, y_{\alpha}, T\right):=\left(x_{\alpha}^{0}, \ldots, x_{\alpha}^{n-k}, y_{\alpha}^{1}, \ldots, y_{\alpha}^{k-1},\left[t^{0}: \cdots: t^{n-k}\right]\right)\right\},
$$

and the blowing-up, in these local coordinates, is given by

$$
\widetilde{U_{\alpha}}=\left\{\left(x_{\alpha}, y_{\alpha}, T\right) \mid x_{\alpha}^{i} t^{j}-x_{\alpha}^{j} t^{i}=0 \quad \text { for } i, j=0 \ldots, n-k\right\} .
$$

Then, we have for example

$$
\sigma\left(x_{\alpha}^{0}, t^{0}, t^{1}, \ldots, t^{n-k}, y_{\alpha}^{1}, \ldots, y_{\alpha}^{k-1}\right)=\left(x_{\alpha}^{0}, x_{\alpha}^{0} t^{1}, x_{\alpha}^{0} t^{2}, \ldots, x_{\alpha}^{0} t^{n-k}, y_{\alpha}^{1}, \ldots, y_{\alpha}^{k-1}\right),
$$

and then

$$
\sigma^{*}(\eta)=-\left(x_{\alpha}^{0}\right)^{n-k+1} \widetilde{\eta}, \quad \widetilde{\eta}=d t^{1} \wedge \cdots \wedge d t^{n-k}
$$

where $\left\{x_{\alpha}^{0}=0\right\}$ respresented the local equation of the exceptional divisor $E$ and $\widetilde{\eta}$ induces $\widetilde{\mathcal{F}}$, the strict transformation of the foliation.

From this equation, we see that the strict transformed foliation $\widetilde{\mathcal{F}}$ is transversal to the exceptional divisor andy it defines the projective flat connection on $\mathbb{P}(\nu(K, M))$ andy the foliation $\widetilde{\mathcal{F}}$ on the open set

$$
\widetilde{U}=\bigcup_{\alpha} \widetilde{U_{\alpha}}
$$

in non singular and defined by a representation $\rho: \pi_{1}(K, *) \rightarrow \mathbf{P G L}(n-k, \mathbb{C})$. So, it has a projective transverse structure.

Transversally projective foliations, the structure is defined by the Maurer-Cartan forms. Therefore, on a neighborhood $U$ we have a family of meromorphic 1 -forms for this structure.

Following [17, pag- 132], as a homogeneous space, the projective space $\mathbb{P}^{n-k}$, is $\mathcal{L} / \mathcal{L}_{0}$ where

$$
\begin{aligned}
\mathcal{L} & =\mathbf{P G L}(n-k, \mathbb{C})=\mathbf{S L}(n-k+1, \mathbb{C}) / \text { center } \\
\mathcal{L}_{0} & =\left\{\left(\begin{array}{ll}
\mathbb{A} & 0 \\
\mathbf{v} & a
\end{array}\right) \in \mathbf{S L}(n-k+1, \mathbb{C})\right\} / \text { center }
\end{aligned}
$$

where $\mathbb{A} \in \mathbf{G L}(n-k, \mathbb{C}), \quad \mathbf{v} \in \mathbb{C}^{n-k}$ is a row $(n-k)$-vector, $\operatorname{tr}(\mathbb{A})=-a$,

$$
\mathcal{L}_{1}\left\{\left(\begin{array}{cc}
\mathbb{I}_{n} & 0 \\
\mathbf{v} & 1
\end{array}\right) \| \mathbf{v} \in \mathbb{C}^{n} \text { row vector }\right\} .
$$

The graded Lie Algebra $\mathfrak{l}=\mathfrak{g}_{-1}+\mathfrak{g}_{0}+\mathfrak{g}_{1}$ with this $\mathcal{L} / \mathcal{L}_{0}$, given by $\mathfrak{l}=\mathfrak{s l}(n+1, \mathbb{C})$ andy

$$
\mathfrak{g}_{-1}=\left\{\left(\begin{array}{ll}
0 & \mathbf{v} \\
\mathbf{0} & 0
\end{array}\right)\right\} \quad \mathfrak{g}_{0}=\left\{\left(\begin{array}{cc}
\mathbb{A} & \mathbf{0} \\
\mathbf{0} & a
\end{array}\right)\right\} \quad \mathfrak{g}_{1}=\left\{\left(\begin{array}{ll}
\mathbf{0} & \mathbf{0} \\
\mathbf{v} & 0
\end{array}\right)\right\}
$$

The invariant forms, are denoted by

$$
\left\{\omega^{i}, \omega_{i}^{j}, \omega_{j} \| i, j=1, \ldots n-k\right\}
$$

and the structure equations are

$$
\begin{aligned}
d \omega^{i} & =-\sum \omega_{k}^{i} \wedge \omega^{k} \\
d \omega_{j}^{i} & =-\sum \omega_{k}^{i} \wedge \omega_{j}^{k}-\omega^{i} \wedge \omega_{j}+\delta_{j}^{i} \sum \omega_{k} \wedge \omega^{k} \\
d \omega_{j} & =-\sum \omega_{k} \wedge \omega_{j}^{k},
\end{aligned}
$$


For the projective space $\mathbb{P}^{1}$, the above equation looks like this

$$
\begin{aligned}
d \omega_{1} & =\omega_{0} \wedge \omega^{1}, \\
d \omega_{0} & =\omega^{1} \wedge \omega_{1}, \\
d \omega^{1} & =\omega_{1} \wedge \omega_{0} .
\end{aligned}
$$

Therefore, on a neighborhood of the Kupka set, there is a family of meromorphic 1 -forms

$$
\left\{\Omega_{i}, \Omega_{i}^{j}, \Omega^{j} \| i, j=1, \ldots, n-k\right\}
$$

obtained by the projection of the holomorphic 1 -forms on the neighborhood of the exceptional divisor.

\section{BAum-Bott Theory.}

Let us recall the results of [4] and 24] in a general context. We need some preliminary notions.

A polynomial invariant function $F: M_{n \times n}(\mathbb{C}) \rightarrow \mathbb{C}$, is a function which can be expressed as a complex polynomial in the entries of the matrix, and satisfies

$$
F(\mathbb{A})=F\left(\mathbf{g} \cdot \mathbb{A} \cdot \mathbf{g}^{-1}\right)
$$

for all $\mathbb{A} \in M_{n \times n}(\mathbb{C})$ and $\mathbf{g} \in G L(n, \mathbb{C})$. It follows that $F$ define a function on $\operatorname{End}(V) \rightarrow \mathbb{C}$, for some complex vector space of dimension $n$.

The main example of a polynomial invariant functions, are the coefficients of

$$
\operatorname{det}(\mathbb{I}+t \mathbb{A})=1+c_{1}(\mathbb{A}) t+\cdots+c_{n}(\mathbb{A}) t^{n},
$$

Observe that $c_{1}(\mathbb{A})=\operatorname{tr}(\mathbb{A})$ and $c_{n}(\mathbb{A})=\operatorname{det}(\mathbb{A})$.

Now, Let $\varphi \in \mathbb{C}\left[X_{1}, \ldots, X_{n}\right]$ be a symmetric and homogeneous of degree $n$. It defines an invariant function if it can be expressed as

$$
\varphi\left(X_{1}, \ldots, X_{n}\right)=\widetilde{\varphi}\left(c_{1}(X), \ldots, c_{n}(X)\right), \quad X=\left(X_{1}, \ldots, X_{n}\right)
$$

for some polynomial $\widetilde{\varphi}$ and considering the variables as the spectrum of a matrix.

Now, consider a 1 dimensional holomorphic foliation $\mathcal{F}$ on a compact complex manifold $M$ of complex dimansion $n$. Such a foliation, is represented by a class $\mathbf{X} \in H^{0}(M, \Theta(L))$, where $L$ is a line bundle on $M$.

In this case, the tangent sheaf of $\mathcal{F}$ is the line bundle $L^{*}$ and the normal sheaf $N_{\mathcal{F}}$ may be considered as the virtual bundle $T M-L$.

Assume that the singular set $S(\mathcal{F})$ is a finite set of points. Now, for any singular point $p \in S(\mathcal{F})$, we take $z=\left(z_{1}, \ldots, z_{n}\right)$ a coordinate system around a singular point $p \in S(\mathcal{F})$ such that $z(p)=0$, and

$$
\mathbf{X}(z)=\sum_{i=1}^{n} X_{i}(z) \frac{\partial}{\partial z_{i}}, \quad X_{i}(0)=0, \quad J_{\mathbf{X}}(z)=\left(\frac{\partial X_{i}}{\partial z_{j}}(z)\right)
$$

be a holomorphic vector field representing $\mathcal{F}$ in a neighborhood of $z(p)=0$.

The Grothendieck point residue

$$
\operatorname{Res}\left(\varphi\left(J_{\mathbf{X}}\right), \mathcal{F}, p\right)=\left(\frac{1}{2 \pi i}\right)^{n} \int_{\Gamma_{\epsilon}} \frac{\varphi\left(J_{\mathbf{X}}\right) d z_{1} \wedge \ldots \wedge d z_{n}}{X_{1}(z) \ldots X_{n}(z)},
$$

where integration is over the $n$-cycle

$$
\Gamma_{\epsilon}=\left\{z:\left|X_{i}(z)\right|=\varepsilon_{i}, 1 \leq i \leq n\right\}, \quad \epsilon=\left(\varepsilon_{1}, \ldots, \varepsilon_{n}\right) \in \mathbb{R}_{>0}^{n}, \quad|\epsilon|<<1,
$$

oriented by declaring positive the form $d\left(\arg f_{1}\right) \wedge \ldots \wedge d\left(\arg f_{n}\right)$.

It is not difficult see that this residue independent of the vector field representing $\mathcal{F}$ around $p$. We have the following. 
Theorem 3.1 (Baum-Bott [4]). Let $M$ be a compact complex manifold of complex dimension $n$. Let $\mathcal{F}$ be a holomorphic foliation by curves on $M$, represented by a meromorphic vector field $\mathbf{X}$. Then

$$
\varphi\left(N_{\mathcal{F}}\right)[M]=\sum_{p \in S(\mathcal{F})} \operatorname{Res}\left(\varphi\left(J_{X}\right), \mathcal{F}, p\right)
$$

For higher dimensional holomorphic foliations, there is also a residue theorem: let $\mathcal{F}$ be a $k$-dimensional holomorphic foliation on an $n$ dimensional complex manifold $M$. Let $Z$ be a connected component of the singular set $S(\mathcal{F})$. We consider the sequence of homomorphism

$$
H_{j}(Z, \mathbb{C}) \stackrel{\jmath_{*}}{\rightarrow} H_{j}(M, \mathbb{C}) \stackrel{\rho}{\rightarrow} H^{2 n-j}(M, \mathbb{C}) \quad j=0,1, \ldots, 2 n,
$$

where $\jmath_{*}$ is induced by inclusion and $\rho$ is the Poincaré duality isomorphism. Set $\alpha_{*}=\rho \circ \jmath_{*}$.

Theorem 3.2 (Baum-Bott [4]). Let $\mathcal{F}$ be a foliation of $\operatorname{dim}(\mathcal{F})=k$ on a manifold $M$ of complex dimension $n$. Let $\varphi \in \mathbb{C}\left[X_{1}, \ldots, X_{n}\right]$ be symmetric and homogenous of degree $r$, with $n-k<r \leq n$, and $Z$ be a connected component of $S(\mathcal{F})$. Then there exists a homology class $\operatorname{Res}(\varphi, \mathcal{F}, Z) \in H_{2 n-2 r}(Z, \mathbb{C})$ such that

(1) $\operatorname{Res}(\varphi, \mathcal{F}, Z)$ depends only on $\varphi$ and on the local behavior of the leaves of $\mathcal{F}$ near $Z$.

(2) $\varphi\left(N_{\mathcal{F}}\right)=\sum_{Z} \alpha_{*} \operatorname{Res}(\varphi, \mathcal{F}, Z)$.

We will use the above theorem in the case in that $\operatorname{deg}(\varphi)=n-k+1$. The fact that $\operatorname{dim}_{\mathbb{C}} S(\mathcal{F}) \leq k-1$, implies that only the components of dimension $k-1$ of $S(\mathcal{F})$ intervene. This is because, $\operatorname{since} \operatorname{Res}(\varphi, \mathcal{F}, Z) \in H_{2 k-2}(Z, \mathbb{C})$, components of dimension smaller than $k-1$ contribute nothing.

Theorem 3.3. Let $\mathcal{F} \in \mathcal{K}_{k}(M, L)$ be a foliation with a compact connected component $K$ and transversal type $\mathbf{X}$. Then

$$
\left.[K] \cdot \operatorname{Res}\left(c_{1}\left(J_{\mathbf{X}}\right)\right)^{\operatorname{cod}(\mathcal{F})+1}, \mathbf{X}, 0\right)=c_{1}\left(N_{\mathcal{F}}\right)^{\operatorname{cod}(\mathcal{F})+1} .
$$

Proof. Suppose that $S_{k-1}(\mathcal{F})=K(\mathcal{F})$, where $K(\mathcal{F})$ compact connected Kupka set of transversal type

$$
\mathbf{X}\left(x_{0}, \ldots, x_{n-k}\right)=\sum_{i=0}^{n-k} X_{i}\left(x_{0}, \ldots, x_{n-k}\right) \frac{\partial}{\partial x_{i}}, \quad \operatorname{cod}(\mathcal{F})=n-k
$$

Set $\varphi=c_{1}^{n-k+1}\left(N_{\mathcal{F}}\right)$. We have

$$
c_{1}^{n-k+1}\left(N_{\mathcal{F}}\right)=\operatorname{Res}\left(c_{1}^{n-k+1}, \mathcal{F}, K(\mathcal{F})\right)[K(\mathcal{F})],
$$

by Theorem 3.2 , but

$$
\operatorname{Res}\left(c_{1}^{n-k+1}, \mathcal{F}, K(\mathcal{F})\right)=\left(\frac{1}{2 \pi i}\right)^{n-k+1} \int_{\Gamma_{\epsilon}} \frac{c_{1}^{n-k+1}\left(J_{\mathbf{X}}(0)\right) d x_{0} \wedge \ldots \wedge d x_{n-k}}{X_{0}(x) \cdots X_{n-k}(x)}
$$

where integration is over the $n-k+1$-cycle $\Gamma_{\epsilon}=\left\{z:\left|X_{i}(z)\right|=\varepsilon_{i}, 1 \leq i \leq n\right\}$, $\varepsilon=\left(\varepsilon_{1}, \ldots, \varepsilon_{n}\right) \in \mathbb{R}_{>0}^{n},|\varepsilon|<<1$. 


\section{Holomorphic Foliations on the Projective SPACE.}

4.1. The projective space. Recall the Euler sequence

$$
0 \longrightarrow \mathcal{O}_{n}(-1) \longrightarrow \bigoplus^{n+1} \mathcal{O}_{n} \longrightarrow \Theta_{n}(-1) \longrightarrow 0
$$

after taking the dual the $(n-k)$ exterior power, we get the exact sequence

$$
0 \longrightarrow \Omega_{n}^{n-k}(n-k) \longrightarrow \bigwedge^{n-k}\left(\bigoplus^{n+1} \mathcal{O}_{n}\right) \approx \bigoplus^{\left(\begin{array}{c}
n+1 \\
n-k
\end{array}\right)} \mathcal{O}_{n} \longrightarrow \Omega_{n}^{n-k-1}(n-k) \longrightarrow 0
$$

As a consequence, a global section $\Omega$ of the sheaf $\Omega_{n}^{n-k}(c)$, may be thought as a polynomial $(n-k)$-form on $\mathbb{C}^{n+1}$ with homogeneous coefficients of degree $\operatorname{deg}_{h}(\Omega)=c-n+k$, which we will still denote by $\Omega$ and satisfying

$$
\imath_{\mathcal{R}} \Omega=0, \quad \text { where } \quad \mathcal{R}=x_{0} \frac{\partial}{\partial x_{0}}+\cdots+x_{n} \frac{\partial}{\partial x_{n}}
$$

is the radial vector field on $\mathbb{C}^{n+1}$. Consequently, the space of sections $\left.H^{0}\left(\mathbb{P}^{n}, \Omega_{n}^{n-k}(c)\right)\right)$, has dimension

$$
h^{0}\left(\mathbb{P}^{n}, \Omega_{n}^{n-k}(c)\right)=\left(\begin{array}{c}
c+k \\
c
\end{array}\right) \cdot\left(\begin{array}{c}
c-1 \\
n-k
\end{array}\right), \quad c \geq n-k+1
$$

and 0 otherwise. Therefore, only the classes $c \geq n-k+1$ could carried foliations of dimension $k$.

Now, we introduce the notion of degree of a foliation of the projective space. Let $\mathcal{F}$ be represented by a section $\Omega \in H^{0}\left(\mathbb{P}^{n}, \Omega_{n}^{n-k}(c)\right)$. We also assume that $\operatorname{dim}_{\mathbb{C}} S(\mathcal{F}) \leq k-1$. Let $\ell: \mathbb{P}^{n-k} \hookrightarrow \mathbb{P}^{n}$ be a linear generic immersion with respect to $\mathcal{F}$, in particular, $\ell\left(\mathbb{P}^{n-k}\right)$ does not hit the singular set. Now, consider the pullback form $\ell^{*}(\Omega) \in H^{0}\left(\mathbb{P}^{n-k}, \Omega_{n-k}^{n-k}(c)\right)$. We observe that $\Omega_{n-k}^{n-k}(c)=\mathcal{K}_{n-k}(c)$, is a holomorphic line bundle, so that $\mathcal{K}_{n-k}(c)=\mathcal{O}_{n-k}(d)$ for some $d \in \mathbb{Z}$. Since $\mathcal{K}_{n-k}=\mathcal{O}_{n-k}(-(n-k+1))$, we get $d=c-(n-k+1)$.

The zero divisor $\left\{\ell^{*}(\Omega)=0\right\}$, reflects the set of tangency between $\mathcal{F}$ and the linear subspace $\ell\left(\mathbb{P}^{n-k}\right) \simeq \mathbb{P}^{n-k} \subset \mathbb{P}^{n}$. The degree of the foliation $\mathcal{F}$ denoted by $\operatorname{deg}(\mathcal{F})$, is the degree of such tangency divisor. By definition

$$
\operatorname{deg}(\mathcal{F})=c_{1}\left(N_{\mathcal{F}}\right)-\operatorname{cod}(\mathcal{F})-1=c_{1}\left(N_{\mathcal{F}}\right)+\operatorname{dim}(\mathcal{F})-n-1 .
$$

Therefore, for foliations on the projective space, we have three discrete invariants: The first Chern class of the normal bundle $c_{1}\left(N_{\mathcal{F}}\right)$ (or topological degree), the degree $\operatorname{deg}(\mathcal{F})$ and the homogeneous degree $\operatorname{deg}_{h}(\mathcal{F})$ andy related by the formulaes

$$
\begin{aligned}
c_{1}\left(N_{\mathcal{F}}\right) & =\operatorname{deg}(\mathcal{F})+\operatorname{cod}(\mathcal{F})+1, \\
\operatorname{deg}_{h}(\mathcal{F}) & =c_{1}\left(N_{\mathcal{F}}\right)-\operatorname{cod}(\mathcal{F})=\operatorname{deg}(\mathcal{F})+1,
\end{aligned}
$$

For instance, for any $n \geq 2$ and for any codimension one holomorphic foliation $\mathcal{F}$ on the projective space $\mathbb{P}^{n}$, we have the formula $c_{1}\left(N_{\mathcal{F}}\right)=d_{h}(\mathcal{F})+1=\operatorname{deg}(\mathcal{F})+2$.

The example of a foliation $\mathcal{F} \in \mathcal{F}_{k}(n, n-k)$ and degree $\operatorname{deg}(\mathcal{F})=0$ is

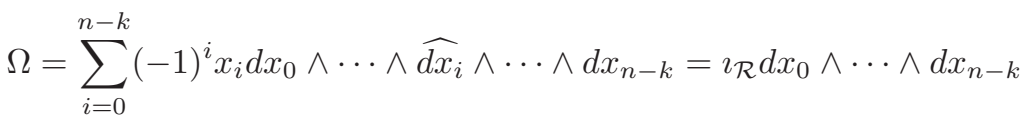

whose leaves are the fibers of the linear fibration

$$
\Phi\left(z_{0}: \cdots: z_{n}\right)=\left[z_{0}: \cdots: z_{n-k}\right]: \mathbb{P}^{n}-\rightarrow \mathbb{P}^{n-k} .
$$

It is well known that the singular set of a codimension one foliation $\mathcal{F}$ in $\mathbb{P}^{n}$, has at least an irreducible component in $S_{n-2}(\mathcal{F})$ (see [19]). Recently, Corrêa Jr- 
Fernández Pérez 12 proved that it is valid for foliations of arbitrary dimension on projective manifolds.

Theorem 4.1. Let $\mathcal{F} \in \mathcal{F}_{k}(M, L)$ be a holomorphic foliation such that $\operatorname{dim}_{\mathbb{C}} S(\mathcal{F}) \leq$ $k-1$. Suppose that $L$ is ample, then $S_{k-1}(\mathcal{F}) \neq \emptyset$

Remark 4.2. The hypotheses of the ampleness of the line bundle $L=\operatorname{det}\left(N_{\mathcal{F}}\right)$ is not restrictive. This hypothesis is always satisfy for foliations on the projective space.

4.2. Foliations with a Kupka component. We give the main examples of $k$ dimensional holomorphic foliations $\mathcal{F}$ with a compact Kupka component on the projective space.

Example 4.3. Let $\left\{f_{0}, \ldots, f_{n-k}\right\}, \quad 1<k<n$, be a collection of homogenous polynomials in $\mathbb{C}^{n+1}$ of degree $\operatorname{deg}\left(f_{j}\right)=d_{j}$.

We assume that $\left\{f:=f_{0} \cdots f_{n-k}=0\right\}$ is a normal crossings divisor of degree $c=d_{0}+\cdots+d_{n-k}$. In other words, for all $j=0, \ldots n-k$, we have holomorphic sections $f_{j} \in H^{0}\left(\mathbb{P}^{n}, \mathcal{O}_{n}\left(d_{j}\right)\right)$ andy $f \in H^{0}\left(\mathbb{P}^{n}, \mathcal{O}_{n}(c)\right)$.

Consider the homogeneous $(n-k)$-form in $\mathbb{C}^{n+1}$ defined by

$$
\begin{aligned}
\Omega\left(z_{0}, \ldots, z_{n}\right) & =\sum_{j=0}^{n-k}(-1)^{j} d_{j} \cdot f_{j}\left(z_{0}, \ldots, z_{n}\right) d f_{0} \wedge \cdots \wedge \widehat{d f}_{j} \wedge \cdots \wedge d f_{n-k} \\
& =\left(f_{0} \ldots f_{n-k}\right) \sum_{j=0}^{n-k}(-1)^{j} d_{j}\left\{\frac{d f_{0}}{f_{0}} \wedge \cdots \frac{\widehat{d f_{j}}}{f_{j}} \cdots \wedge \frac{d f_{n-k}}{f_{n-k}}\right\} .
\end{aligned}
$$

The homogeneous $(n-k)$-form $\Omega$ has degree $c-n+k$. It vanishes the radial vector field $\mathcal{R}$. In fact, we observe that $\Omega=\imath_{\mathcal{R}} d f_{0} \wedge \cdots d f_{n-k}$, the contraction by the radial vector field $\mathcal{R}$. Moreover, the $(n-k)$-form $\Omega$, defines a foliation $\mathcal{F} \in \mathcal{F}_{k}(n, c)$, whose leaves are the fibers of the rational ramified fibration

$$
\begin{aligned}
\Phi: \mathbb{P}^{n} & -\rightarrow \mathbb{P}^{n-k} \\
z & \mapsto\left[f_{0}^{m_{0}}(z): \cdots: f_{n-k}^{m_{n-k}}(z)\right]
\end{aligned}
$$

where $\left\{m_{0}, \ldots, m_{n-k}\right\}$ are integers relatively prime satisfying the equations

$$
m_{0} d_{0}=\cdots=m_{n-k} d_{n-k}=d \text { for some } \quad d \in \mathbb{N} .
$$

The foliation belongs to the set $\mathcal{K}_{k}(n, c)$. The Kupka set is the complete intersection $K=\left\{f_{0}=\cdots=f_{n-k}=0\right\}$. This is also the indetermination locus of the rational map $\Phi: \mathbb{P}^{n} \rightarrow \mathbb{P}^{n-k}$.

It is clear that the set $\left\{f_{0}=\cdots=f_{n-k}=0\right\} \subset S(\Omega)$. Now, consider any $x \in\left\{f_{0}=\cdots=f_{n-k}=0\right\}$ and a direct calculation gives

$$
d \Omega(x)=\left(\sum_{j=0}^{n-k} d_{j}\right) d f_{0} \wedge \cdots \wedge d f_{n-k}(x)=c d f_{0} \wedge \cdots \wedge d f_{n-k}(x) \neq 0,
$$

this last inequality, follows from the transversally condition. The submanifold $K$ has degree $\operatorname{deg}(K)=d_{0} \cdots d_{n-k}$.

Finally, we observe that the transversal type of the Kupka component is the linear vector field

$$
\mathbf{X}\left(x_{0}, \ldots, x_{n-k}\right)=\sum_{j=0}^{n-k} d_{j} \cdot x_{j} \frac{\partial}{\partial x_{j}}
$$


The foliations described above are called rational components. They are irreducible components of the space of foliations if $2 \leq k<n$ [13]. We denote them by

$$
\mathcal{R}_{k}\left(n: d_{0}, \ldots, d_{n-k}\right) \subset \mathcal{F}_{k}(n, c), \quad \text { where } \quad c=\sum_{j=0}^{n-k} d_{j} .
$$

Observe that the rational component has $n-k+1$ discrete parameters, the degree of the divisors involved $\left(d_{0}, \ldots, d_{n-k}\right) \in \mathbb{N}^{n-k+1}$, which are related with the first Chern class of the normal sheaf of the foliation and the degree of its Kupka component.

In the codimension one case, there is a complete classification of the set $K_{n-1}(n, c)$ when $n \geq 3$. (see Brunella [5] and Calvo-Andrade [6, 7]).

Theorem 4.4. The set $\mathcal{K}_{n-1}(n, c, d)=\left\{\mathcal{F} \in \mathcal{K}_{n-1}(n, c) \mid \operatorname{deg}(K)=d\right\} \neq \emptyset$ if and only if the system of equations

$$
x \cdot y=d \quad x+y=c
$$

has positive integers as solution, say $(a, b) \in \mathbb{N} \times \mathbb{N}$. In this case, we have

$$
\mathcal{K}_{n-1}(n, c, d)=\mathcal{R}_{n-1}(n: a, b) .
$$

For any $\mathbb{Z} \ni c \geq 2$ the set $\mathcal{K}_{n-1}(n, c)$ has $[c / 2]$ irreducible components. In the codimension one case, the transversal type is always of the type

$$
\mathbf{X}_{p q}=p x \frac{\partial}{\partial x}+q y \frac{\partial}{\partial y} \quad p, q \in \mathbb{N}, \quad \text { where } \begin{cases}\text { radial } & p=q=1 \\ \text { non-resonant } & (p, q)=1 \\ \text { resonant } & 1=p<q\end{cases}
$$

and we get $a=p c / p+q$ and $b=q c / p+q$.

An important intermediate problem, towards the classification of the $k$ dimensional foliations with a Kupka component on the projective space, is the following.

Problem 4.5. Given $\mathcal{F} \in \mathcal{K}_{k}(n, c)$. Classify all the possible transversal type of its Kupka component.

\section{Rational Fibrations.}

5.1. Geometrical Preliminary results. We begin this section, with some results on the projective space.

There are two classes of theorems which are of our interest about submanifolds of a given manifold $M$ :

Given $X \subset M$ a smooth submanifold and let $X \subset U \subset M$ be an open neighborhood of $X$. The property that we need is that any meromorphic object defined in $U$, may be extended to an object on $M$.

The first results in this direction is the following.

Theorem 5.1 ([2], [22]). Let $Y$ be a connected analytic subset of $\mathbb{P}^{n}, \quad n \geq 2$, with $\operatorname{dim} Y \geq 1$. Then any meromorphic function in a connected neighborhood of $Y$ extends to a meromorphic function on all of $\mathbb{P}^{n}$.

On the other hand, given a smooth submanifold $X \subset \mathbb{P}^{n}$, the normal bundle $\nu\left(X, \mathbb{P}^{n}\right)$ impose some conditions on the embedding of $X \hookrightarrow \mathbb{P}^{n}$. In this direction, there are some results that we are going to use.

Theorem 5.2 ([1]). Let $X \subset \mathbb{P}^{n}$ be a smooth submanifold. Assume that $\operatorname{dim}(X)=$ $d \geq 3$ and $n=2 d-1$. If the normal bundle $\nu\left(X, \mathbb{P}^{n}\right)$ decompose as a direct sum of line bundles, then $\operatorname{Pic}(X)=\mathbb{Z} \cdot\left[\mathcal{O}_{X}(1)\right]$ 
It follows from this result, that the normal bundle extends to $\mathbb{P}^{n}$ and $X$ is a complete intersection.

Theorem 1. Let $\mathcal{F} \in \mathcal{K}_{k}(n, c)$ be a holomorphic foliation with $S_{k-1}(\mathcal{F})=K(\mathcal{F})$ and transversal type

$$
\mathbf{X}=\sum_{i=0}^{n-k} \lambda_{i} x_{i} \frac{\partial}{\partial x_{i}}, \quad \lambda_{i} \in \mathbb{N} \quad \lambda_{i} \neq \lambda_{j}, \text { for all } i \neq j
$$

Then the leaves of the foliation are the fibers of a rational fibration and it is represented by a $(n-k)$-form of the type

$$
\Omega\left(z_{0}, \ldots, z_{n}\right)=\sum_{i=0}^{n-k}(-1)^{i} \lambda_{i} \cdot f_{i}\left(z_{0}, \ldots, z_{n}\right) d f_{0} \wedge \ldots \wedge \widehat{d f_{i}} \wedge \ldots \wedge d f_{n-k} .
$$

Proof. We use the Theorems 2.17 and 2.18 of the section 2 .

Let $\Lambda_{N R}=\left\{\lambda_{0}<\cdots \lambda_{\ell}\right\}$ and $\Lambda_{R}=\left\{\lambda_{\ell+1}<\cdots<\lambda_{n-k}\right\}$ the resonant and non-resonant part respectively. By (2.17) and (2.18), on a neighborhood of the Kupka set, we have the integrating factor $G=G\left(x_{0}, \ldots, x_{\ell}\right)$. It is a section on $U$ of the line bundle $\operatorname{det}\left(N_{\mathcal{F}}\right)=\mathcal{O}(c)$. Moreover, it can be extended to the projective space by Theorem 5.1 .

Now, the closed meromorphic forms $\theta_{\ell+j}$ are locally, logarithmic differentials of meromorphic functions.

Let $f_{j} \in H^{0}\left(\mathbb{P}^{n}, \mathcal{O}\left(d_{j}\right)\right)$ be the extension of the components of the invariant divisor on $U$. We also consider the extension of the rational functions

$$
\bar{\varphi}^{\ell+k}=\frac{f_{\ell+k}}{f_{0}^{m_{0}^{\ell+k}} \cdots f_{\ell}^{m_{\ell}^{\ell+k}}}
$$

Let $g_{\ell+k}=f_{\ell+k}+c_{\ell+k} \cdot f_{0}^{m_{0}^{\ell+k}} \cdots f_{\ell}^{m_{\ell}^{\ell+k}}$, where $c_{\ell+k} \in \mathbb{C}$. Then the fibration is

$$
\left[f_{0}^{\lambda_{0}}: \cdots: f_{\ell}^{\lambda_{\ell}}: g_{\ell+1}^{\lambda_{\ell+1}}: \cdots: g_{n-k}^{\lambda_{n-k}}\right]: \mathbb{P}^{n} \rightarrow \mathbb{P}^{n-k} .
$$

For the Corollary 2, we begin with some remarks on the Kupka set.

Let $\mathcal{F} \in \mathcal{K}_{k}(M, L)$ with a Kupka component $K$, and transversal type

$$
\mathbf{X}\left(x_{0}, \ldots, x_{n-k}\right)=\sum_{i=0}^{n-k}\left(\lambda_{i} x_{i}+\text { h.o.t }\right) \frac{\partial}{\partial x_{i}},
$$

then, the residue has the expression

$$
\operatorname{Res}\left(c_{1}^{n-k+1}, \mathcal{F}, K(\mathcal{F})\right)=\frac{\left(\sum_{i=0}^{n-k} \lambda_{i}\right)^{n-k+1}}{\prod_{i=0}^{n-k} \lambda_{i}}=\prod_{j=0}^{n-k}\left(\sum_{i=0}^{n-k} \frac{\lambda_{i}}{\lambda_{j}}\right) .
$$

Then, by Theorem 3.3, we have

$$
c_{1}\left(N_{\mathcal{F}}\right)^{n-k+1}=\prod_{j=0}^{n-k}\left(\sum_{i=0}^{n-k} \frac{\lambda_{i}}{\lambda_{j}}\right) \cdot[K] .
$$

Hence we have

$$
[K]=\prod_{i-0}^{n-k}\left(\frac{\lambda_{i} c_{1}\left(N_{\mathcal{F}}\right)}{\sum_{i=0}^{n-k} \lambda_{i}}\right) \in H^{2(n-k+1)}(M, \mathbb{C}) .
$$

In particular, for a foliation $\mathcal{F} \in \mathcal{F}_{k}(n, c)$, the above formula looks as follows 


$$
\operatorname{deg}(K)=\prod_{i=0}^{n-k} \frac{\lambda_{i}(d(\mathcal{F})+\operatorname{cod}(\mathcal{F})+1)}{\sum_{i=0}^{n-k} \lambda_{i}}=\prod_{i=0}^{n-k} \frac{\lambda_{i} c}{\sum_{i=0}^{n-k} \lambda_{i}}
$$

Now, Let $\mathcal{F} \in \mathcal{K}_{k}(n, c)$ be a foliation with a compact Kupka set $K=K(\mathcal{F})$. Let

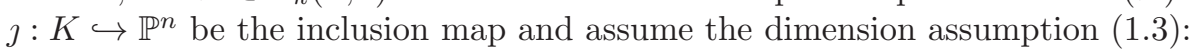

$$
4 \leq \operatorname{dim} \mathcal{F} \quad \operatorname{dim}(\mathcal{F}) \geq \operatorname{cod}(\mathcal{F})+2 .
$$

Since the linear part of the transversal type has different eigenvalues, the normal bundle splits as a direct sum of line bundles associated to the proper directions [8].

$$
\nu\left(K, \mathbb{P}^{n}\right)=\bigoplus_{i=0}^{n-k} L_{i}
$$

Because $K$ is subcanonical and $\nu\left(K, \mathbb{P}^{n}\right)=\mathcal{O}_{K}(c), \quad c=c_{1}\left(N_{\mathcal{F}}\right)$, we have

$$
c_{1}\left(\nu\left(K, \mathbb{P}^{n}\right)=\jmath^{*}\left(c_{1}\left(N_{\mathcal{F}}\right)\right)=\sum_{i=0}^{n-k} c_{1}\left(L_{i}\right) \in H^{2}(K, \mathbb{Z}) .\right.
$$

On the other hand, the classes

$$
\mathbf{c}_{i}:=\left(\frac{\lambda_{i}}{\sum_{i=0}^{n-k} \lambda_{i}}\right) \jmath^{*}\left(c_{1}\left(N_{\mathcal{F}}\right)\right) \in H^{2}(K, \mathbb{Q}), \quad i=0, \ldots, n-k
$$

are precisely the Chern classes $c\left(L_{i}\right)$ of the line bundles $L_{i}$, then these classes are integers and the same the numbers

$$
d_{i}=\frac{\lambda_{i} c}{\sum_{i=0}^{n-k} \lambda_{i}} \in \mathbb{Z} \simeq H^{2}(K, \mathbb{Z})
$$

Then, we have proved.

Corollary 2. Let $\mathcal{F} \in K_{k}(n, c)$ be a foliation of small codimension and linear transversal type

$$
\mathbf{X}=\sum_{i=0}^{k} \lambda_{i} x_{i} \frac{\partial}{\partial x_{i}} \quad \lambda_{i} \neq \lambda_{j} \quad \text { for all } \quad i \neq j
$$

then $\lambda_{i} \in \mathbb{N}$ and $\mathcal{F}$ is a rational fibration.

Now, we consider the radial case.

Theorem 3. Let $\mathcal{F} \in K_{k}(n, c)$ with a Kupka set $K$ and radial transversal type. If $K$ is a complete intersection, then $\mathcal{F}$ is a rational fibration of the type

$$
\left[f_{0}: \cdots: f_{n-k}\right]: \mathbb{P}^{n} \rightarrow \mathbb{P}^{n-k}, \quad \operatorname{deg}\left(f_{j}\right)=\frac{c}{n-k+1}
$$

Proof. Let $U$ be a neighborhood of the Kupka set where the foliation has a projective transverse structure. In this neighborhood we have the Maurer-Cartan forms that defines the structure. We are able to extend this forms and then the foliation has a projective transversal structure on $\mathbb{P}^{n}$.

Since $\mathbb{P}^{n}$ is simply connected, any $\mathbb{P}^{n-k}$ flat bundle is $\mathbb{P}^{n} \times \mathbb{P}^{n-k} \stackrel{\pi_{1}}{\rightarrow} \mathbb{P}^{n}$ and the rational section is of the type $z \rightarrow(z, \Phi(z))$, then $\Phi$ must be a rational fibration. 


\section{An application: Normal FORM of NON-INTEgrable CODimension one} DISTRIBUTIONS.

Let $\omega$ be a germ of 1 -form on $\left(\mathbb{C}^{n}, 0\right)$. We denote by

$$
(d \omega)^{s}=\overbrace{d \omega \wedge \cdots \wedge d \omega}^{\text {s times }} .
$$

Let $\omega \in H^{0}\left(M, \Omega_{M}^{1}(L)\right)$. We define the class of the foliation induced by $\omega$ to be the integer $r$ for which generically

$$
\omega \wedge(d \omega)^{r-1} \neq 0, \quad \omega \wedge(d \omega)^{r} \equiv 0
$$

See 15 for the general theory. by

The Kupka set of the distribution $\mathcal{F}$ induced by $\omega \in H^{0}\left(M, \Omega_{M}^{1}(L)\right)$ is defined

$$
K(\mathcal{F})=\left\{p \in M ; \omega(p)=0,(d \omega)^{r}(p) \neq 0\right\} \subset \operatorname{Sing}(\mathcal{F}) .
$$

The next result gives a Kupka type phenomena in the non integrable case.

Theorem 6.1 (Kupka type phenomena). Let $\omega \in H^{0}\left(M, \Omega_{M}^{1}(L)\right)$ a codimension one holomorphic distribution of class $r$. Given a connected Kupka component $K$ there exists a germ at $0 \in \mathbb{C}^{2 r}$ of a holomorphic 1 -form

$$
\sum_{i=1}^{2 r} A_{i}\left(x_{1}, \ldots, x_{2 r}\right) d x_{i}
$$

with an isolated singularity at $0 \in \mathbb{C}^{2 r}$, an open covering $\left\{U_{\alpha}\right\}_{\alpha}$ of a neighborhood of $K \subset X$, and a family of submersions $\varphi_{\alpha}: U_{\alpha} \rightarrow \mathbb{C}^{2 r}$ such that

$$
\varphi_{\alpha}^{-1}(0)=K \cap U_{\alpha} \quad \text { and } \quad \omega_{\alpha}=\varphi_{\alpha}^{*} \eta .
$$

Proof. Since $(d \omega)^{r}(0) \neq 0$ it follows from the classical Darboux Theorem that

$$
d \omega=\sum_{i=1}^{r} d x_{i} \wedge d x_{i+r} .
$$

Then $d\left(\omega-\sum_{i=1}^{r} x_{i} d x_{i+r}\right)=0$. From Poincaré lemma we get that there exist $f \in \mathcal{O}_{n}$ such that $\omega-\sum_{i=1}^{r} x_{i} d x_{i+r}=d f$. Using that

$$
(d \omega)^{r}=(-1)^{\frac{r(r-1)}{2}} r ! d x_{1} \wedge \cdots \wedge d x_{2 r}
$$

we get

$$
0=\omega \wedge(d \omega)^{r}=(-1)^{\frac{r(r-1)}{2}} r !\left(\sum_{i=1}^{r} x_{i} d x_{i+r}+d f\right) \wedge d x_{1} \wedge \cdots \wedge d x_{2 r} .
$$

Then $d f \wedge d x_{1} \wedge \cdots \wedge d x_{2 r}=0$ implies that

$$
\frac{\partial f}{\partial x_{i}}=0 \text { for all } i=r+1, \ldots, n
$$

i.e., $f$ depends only of the variables $\left(x_{1}, \ldots, x_{2 r}\right)$.

Example 6.2. Let $\mathcal{D}$ be the distribution on $\mathbb{P}^{n}$, of degree $d$, induced given, in homogenous coordinates, by

$$
\omega=\sum_{i=1}^{r-1}\left(f_{i} d f_{i+r}-f_{i+r} d f_{i}\right)
$$

such that $d f_{0} \wedge \cdots \wedge d f_{2 r-1}(z) \neq 0$. The transversal type of $\omega$ is

$$
\omega=\sum_{i=1}^{r-1}\left(x_{i} d x_{i+r}-x_{i+r} d x_{i}\right)
$$


In fact, the map

$$
\left(f_{0}, \ldots, f_{2 r-1}\right): \mathbb{C}^{n+1} \longrightarrow \mathbb{C}^{2 r}
$$

is a submersion, since $d f_{1} \wedge \cdots \wedge d f_{2 r}(z) \neq 0$. Thus, there exist a holomorphic coordinate system $\left(x_{1}, \ldots, x_{2 r}, z\right) \in U \subset \mathbb{C}^{n+1}$ such that $\left.f_{i}\right|_{U}=x_{i}$.

Theorem 4. Let $\mathcal{D}$ be a codimension one distribution on $\mathbb{P}^{n}$, of class $r$, given by a 1 -form $\omega \in H^{0}\left(\mathbb{P}^{n}, \Omega_{\mathbb{P}^{n}}^{1}(d+2)\right)$ such that codSing $\left((d \omega)^{r}\right) \geq 3$. Suppose that the Kupka component of $\mathcal{D}$ has transversal type

$$
\sum_{i=0}^{r-1}\left(x_{i} d x_{i+r}-x_{i+r} d x_{i}\right)
$$

Then $\mathcal{D}$ is induced, in homogenous coordinates, by the 1-form

$$
\sum_{i=0}^{r-1}\left(f_{i} d f_{i+r}-f_{i+r} d f_{i}\right)
$$

Proof. We have that the $(2 r-1)$-form $\Theta=\omega \wedge(d \omega)^{r-1}$ is integrable and $K$ is a Kupka component of radial type of $\Theta$. It follows from Theorem 3 that

$$
\Theta=\omega \wedge(d \omega)^{r-1}=\sum_{i=0}^{2 r-1}(-1)^{i} f_{i} d f_{0} \wedge \cdots \wedge \widehat{d f}_{i} \wedge \cdots \wedge d f_{2 r-1} .
$$

In particular, this implies that $(d \omega)^{r}$ is globally decomposable on $\mathbb{C}^{n+1}$. Since $\operatorname{cod} \operatorname{Sing}\left((d \omega)^{r}\right) \geq 3$ it follows from [10] that

$$
d \omega=\sum_{i=0}^{r-1} d f_{i} \wedge d f_{i+r}
$$

Now, contracting by the radial vector field we conclude

$$
(d+2) \omega=i_{R} d \omega=\sum_{i=0}^{r-1}\left(m_{i+r} f_{i} d f_{i+r}-m_{i} f_{i+r} d f_{i}\right),
$$

where $m_{i}=\operatorname{deg}\left(f_{i}\right)$. Comparing $\omega$ with its transversal type we see that $m_{i}=m_{j}$, for all $i, j=0, \ldots 2 r-1$.

Acknowledgements. We would to thank to the Department of Algebra, geometry, topology and Analysis of the Valladolid University, for its hospitality during the elaboration to this work.

The first author also thank to the Federal University of Minas Gerais UFMG and IMPA, for the hospitality during the elaboration of this work.

O. Calvo-Andrade: Partially supported by $\mathrm{CNPq}$

M. Corrêa Jr.: Partially supported by CAPES-DGU 247/11, CNPq 300352/20123 and PPM-00169-13.

A. Ferrnández-Pérez: Partially supported by CNPq 301635/2013-7 and FAPEMIG APQ-00371-13

\section{REFERENCES}

[1] L. Bădescu: On the normal bundle of submanifolds of $\mathbb{P}^{n}$. Proc. Amer. Math. Soc. 136 (2008), 1505-1513.

[2] W. Barth: Fortsetzung, meromorpher Funktionen in Tori und Komplex projektiven Räumen. Invent. Mat. 5 (1968), 42-62.

[3] B. Basili, C. Peskine: Décomposition du fibré normal des surfaces lisses de $\mathbb{P}^{4}$ et structures doubles sur les solides de $\mathbb{P}^{5}$. Duke Math. J 69 (1993), 87-95.

[4] P. Baum, R. Bott: Singularities of holomorphic foliations. J. Differential Geom. 7 (1972), 279-432. 
[5] M. Brunella: Sur les feuilletages de l'espace projective ayant une composante de Kupka. L'eisegnement mathématique (2) 55 (2009), 1-8.

[6] O. Calvo-Andrade: Foliations with a Kupka component on Algebraic Manifolds. Bol. Soc. Brasil. Mat. vol 302 (1999), 183-197.

[7] O. Calvo-Andrade: Foliations with a radial Kupka set on projective spaces. Available in arXiv:1309.3298. (2008).

[8] O. Calvo-Andrade: Foliations of codimension greater than one with a Kupka component. Qual. Theory Dyn. Syst. 8 (2009), 241-253. doi. 10.1007/s12346-010-0012-y

[9] O. Calvo-Andrade, M. Soares: Chern Numbers of a Kupka component. Ann. L'Institute Fourier. vol 44, fasiculo 4, (1994) pags. 1219-1236

[10] D. Cerveau, Une application du théorème de Frobenius singulier: le théorème de Darboux singulier. C. R. Acad. Sci. Paris Sér. A-B 288 (1979), no. 22, 1021-1024

[11] D. Cerveau, A. Lins Neto: Codimension-one foliations in $\mathbb{C P}(n), n \geq 3$, with Kupka components. Astérisque 222 (1994), 93-132.

[12] M. Corrêa Jr, A. Fernández-Pérez: Minimal sets of higher dimensional foliations on complex projective manifolds. Available in arXiv:1403.4286. (2014).

[13] F. Cukierman, J. V. Pereira, I. Vainsencher. Stability of foliations induced by rational maps. Ann. de la Faculté des Sciences de Toulouse. vol. XVIII, 4 (2009), 685-715.

[14] Fornaess, John Erik; Sibony, Nessim Wold, Erlend Fornaess. Q-Complete domains with corners in $\mathbb{P}^{n}$ and extension of line bundles. Mathematische Zeitschrift 2013.

[15] C. Godbillon, Geometrie differentielle et mecanique analytique, Hermann, 1969.

[16] J. P. Jouanolou, Equations de Pfaff algébriques, Springer LNM, 708 (1979), Springer.

[17] S. Kobayashi: Transformation Groups in Differential Geometry. Ergebnisse der Mathematik und ihrer Grenzgebite, Band 70. Springer-Verlag Berlin Heildelberg New York (1972).

[18] S. Kobayashi, Differential Geometry of Complex Vector Bundles. Kanô Memorial Lectures 5. Princeton University Press. (1987)

[19] A. Lins Neto: Componentes irredutíveis dos espaços de folheações. Publicações Matemáticas do IMPA. Instituto Nacional de Matemática Pura e Aplicada (IMPA), Rio de Janeiro, 2007. $26^{0}$ Colóquio Brasileiro de Matemática.

[20] A. Medeiros: Structural stability of integrable differential forms. In: do Carmo, M. Palis. J. (eds.) Geometry and Topology. Springer LNM 597 (1977), 395-428.

[21] K. Morita: On the basis of twisted de Rham cohomology. Hokkaido Math. J. 27 no. 3 (1998), 567-603.

[22] H. Rossi: Continuation of subvarieties of projective varieties. Amer. J. Math. 91 (1969), 565-575.

[23] B. Scardua, Transversely affine and transversely projective foliations. Ann. Sci. Ecole Norm. Sup. (4) 30 (1997), 169-204.

[24] M. G. Soares: Holomorphic foliations and characteristic numbers. Comm. Contemporary Maths. 7 (5) (2005), 583-596

O. Calvo-Andrade: IMPA. Estrada Dona Castorina 110. Jardim Botânico.

Current address: CIMAT. Apdo. Postal 402, Guanajuato 36000. México.

E-mail address: omegar.mat@gmail.com

M. Corrêa Jr.: Depto. de Mat.-ICEX Universidade Federal de Minas Gerais, UFMG Current address: Av. Antônio Carlos 6627, 31270-901, Belo Horizonte-MG, Brasil.

E-mail address: mauriciomatufmg@gmail.com

A. Fernández-Pérez: Depto. de Mat.-ICEX, Universidade Federal de Minas Gerais, UFMG

Current address: Av. Antônio Carlos 6627, 31270-901, Belo Horizonte-MG, Brasil.

E-mail address: arturofp@mat.ufmg.br 